\title{
LARGE DEVIATIONS OF THE EXIT MEASURE THROUGH A CHARACTERISTIC BOUNDARY FOR A POISSON DRIVEN SDE
}

\author{
Etienne Pardoux* And Brice SAmegni-Kepgnou
}

\begin{abstract}
Let $O$ be the basin of attraction of a given equilibrium of a dynamical system, whose solution is the law of large numbers limit of the solution of a Poissonian SDE as the size of the population tends to $+\infty$. We consider the law of the exit point from $O$ of that Poissonian SDE. We adapt the approach of Day [J. Math. Anal. Appl. 147 (1990) 134-153] who studied the same problem for an ODE with a small Brownian perturbation. For that purpose, we will use the large deviations principle for the Poissonian SDE reflected at the boundary of $O$, studied in our recent work Pardoux and Samegni [Stoch. Anal. Appl. 37 (2019) 836-864]. The main motivation of this work is the extension of the results concerning the time of exit from the set $O$ established in Kratz and Pardoux [Vol. 2215 of Lecture Notes in Math.. Springer (2018) 221-327] and Pardoux and Samegni [J. Appl. Probab. 54 (2017) 905-920] to unbounded open sets $O$. This is done in sections 4.2.5 and 4.2.7 of Britton and Pardoux [Vol. 2255 of Lecture Notes in Math. Springer (2019) 1-120], see also The SIR model with demography subsection below.
\end{abstract}

Mathematics Subject Classification. 60F10, 60H10, 92C60.

Received January 9, 2019. Accepted December 19, 2019.

\section{INTRODUCTION}

We consider a $d$-dimensional process of the type, with any $z \in \mathbb{R}_{+}^{d}$ such that $\sum_{i=1}^{d} z_{i} \leq 1$.

$$
Z^{N}(t)=Z^{N, z}(t):=\frac{[N z]}{N}+\frac{1}{N} \sum_{j=1}^{k} h_{j} P_{j}\left(\int_{0}^{t} N \beta_{j}\left(Z^{N, z}(s)\right) \mathrm{d} s\right) .
$$

Here $\left(P_{j}\right)_{1 \leq j \leq k}$ are i.i.d. standard Poisson processes. The $h_{j} \in \mathbb{Z}^{d}$ denote the $k$ respective jump directions with respective jump rates $\beta_{j}(z)$ and $z \in A$ (where $A$ is the "domain" of the process). In fact (1.1) specifies a continuous time Markov chain with state space

$$
A^{(N)}=\left\{z \in \mathbb{R}_{+}^{d}:\left(N z_{1}, \ldots N z_{d}\right) \in \mathbb{Z}_{+}^{d}, \sum_{i=1}^{d} z_{i} \leq 1\right\} .
$$

Keywords and phrases: Poisson process driven SDE, law of large numbers, large deviations principle.

Aix-Marseille Université, CNRS, Centrale Marseille, I2M, UMR 737313453 Marseille, France.

* Corresponding author: etienne.pardoux@univ-amu.fr 
The following assumption ensures that the process $Z^{N}(t)$ remains in $A^{(N)}$ : for any $1 \leq j \leq k$ such that $h_{j}^{i}<0$ ( $h_{j}^{i}$ denotes the $i$-th coordinate fo the vector $\left.h_{j}\right), \beta_{j}(z)=0$ whenever $z_{i}=0$; moreover, if $\sum_{i=1}^{d} h_{j}^{i}>0, \beta_{j}(z)=0$ whenever $\sum_{i=1}^{d} z_{i}=1$.

We know from the law of large numbers of Kurtz [10], see also Chapter 11 of Ethier and Kurtz [6], and Britton and Pardoux [2], that under mild assumptions on the rates $\beta_{j}, 1 \leq j \leq k$, for all $T>0, Z^{N, z}(t)$ converges to $Y(t, z)$ almost surely and uniformly over the interval $[0, T]$, where $Y(t, z)=Y(t)$ is the solution of the ODE

$$
Y(t, z)=z+\int_{0}^{t} b(Y(s, z)) \mathrm{d} s
$$

with

$$
b(y):=\sum_{j=1}^{k} \beta_{j}(y) h_{j},
$$

and $Y(t, z)$ takes its values in the set

$$
A=\left\{z \in \mathbb{R}_{+}^{d}, \sum_{i=1}^{d} z_{i} \leqslant 1\right\} .
$$

Remark 1.1. Our results would be valid with $A$ replaced by the more general compact set $A_{R}=\{z \in$ $\left.\mathbb{R}_{+}^{d}, \sum_{i=1}^{d} z_{i} \leqslant R\right\}$, and $A^{(N)}$ being redefined accordingly. However, it is essential that $A$ be compact. In fact the extension of the results of [11] to non compact sets relies precisely upon the result of the present paper, as we shall explain in Section 6.3.

We assume that $O$ is the basin of attraction of a stable equilibrium $z^{*}$ of (1.3), in the sense that, starting from any point in the open set $O$, the solution of (1.3) converges to $z^{*}$ as $t \rightarrow \infty$, but this need not be the case when starting from at least part of the boundary of $O$, and there can be other equilibria outside $O$. For the models we have in mind (see the four examples in Sect. 6), $O$ has a characteristic boundary which is either the set $\left\{z \in A ; z_{1}=0\right\}$, or else the part of the boundary of $O$ which is included in $\AA$, the interior of $A$. In both cases, that characteristic boundary is defined as

$$
\widetilde{\partial O}:=\{z \in \partial O ;<b(z), n(z)>:=0\},
$$

where $\langle\cdot, \cdot\rangle$ denotes the scalar product in $\mathbb{R}^{d}$ and $n(z)$ the unit outward normal to $\widetilde{\partial O}$ at $z$. Clearly the solution of the dynamical system (1.3) starting from $z \in \widetilde{\partial O}$ remains in $\widetilde{\partial O}$ for all time. In the example treated in section 6, either $O=\AA$, in which case $\widetilde{\partial O}=\left\{z \in A ; z_{1}=0\right\} \varsubsetneqq \partial O$ (this is the situation treated in Sects. 6.2 and 6.3), or else $A$ is the union of the basins of attraction of both the endemic and the disease free equilibria, and the boundary between them, which is precisely $\widetilde{\partial O}$, this is the situation in both Sections 6.4 and 6.5 , see Figures 1 and 2 in those sections.

Our goal in this paper is to study the behaviour for large $N$ of the exit measure of $Z^{N, z}$ from $O$. In other words, the aim is to study the large $N$ asymptotic of the probability that a trajectory of $Z^{N}$ exits $O$ in the neighborhood of a given point. Our main result, Theorem 5.3, says that, $\tau_{O}^{N}$ denoting the first time that the process $Z^{N, z}(t)$ either exits $\bar{O}$ or hits $\widetilde{\partial O}$, if $y \in \widetilde{\partial O}$ and $\delta>0$, for any $\eta>0$ and $N$ large enough,

$$
\exp \left\{-N\left(S_{z}(y)+\eta\right)\right\} \leq \mathbb{P}_{z}\left(\left|Z^{N}\left(\tau^{N}(O)\right)-y\right|<\delta\right) \leq \exp \left\{-N\left(S_{z}(y)-\eta\right)\right\},
$$

where $S_{z}(y)$ is defined by (5.12).

Our main motivation comes from epidemics models, for which we have already studied a similar asymptotic for the exit time from the same set by the same process in $[8,11]$. Those results are established when $A$ is a 
compact set. However, for the purpose of certain epidemics models, we need to extend those results to a non compact set $A \subset \mathbb{R}_{+}^{d}$, see Section 6.3. This is easily done with the help of Theorem 5.3 of the present paper, once we show that $\inf _{|y| \geq R} S_{z}(y) \rightarrow \infty$ as $R \rightarrow \infty$, uniformly for $z$ in the vicinity of $z^{*}$, see sections 4.2 .5 and 4.2 .7 of Britton and Pardoux [2], and Section 6.3. This is the main expected outcome of the results of the present paper.

Another motivation is to identify, when it exists, the most probable exit neighborhood for the process. It turns out that we can do so in four epidemics models.

As far as we know, this had never been done up to now for Poisson driven SDEs. Similar results have been established for dynamical systems with small Brownian type perturbations, see e.g. chapter 5 of Dembo and Zeitouni [4], which, for the analog of the results we aim at, refer to the work of Day [3].

Our method of proof consists in adapting the approach developed by Day [3]. First we define a reflected Poissonian SDE for which the large deviations principle is satisfied, with the same rate function as the original one defined by (1.1), as has been proved in our earlier paper [12]. We then mimic the arguments of Day [3] to obtain our results.

The identification of a preferred exit neighborhood in the four considered models relies upon the characterization of the quasi-potential as the value function of a deterministic optimal control problem, and the use of the Pontryagin maximum principle to specify as much as possible the optimal trajectory. It turns out that the preferred point $\bar{z}$ happens to be in our examples the unique limit point of the solution of the ODE, when starting from a point on the boundary $\widetilde{\partial O}$.

Let us explain the application to epidemics models. In all our examples, the first coordinate $z_{1}$ will denote the proportion ${ }^{1}$ of susceptible individuals in the population. In the two examples treated in Sections 6.2 and 6.3, for the considered range of values of the parameters, the ODE (1.3) has two equilibria, one stable, the endemic equilibrium $z^{*}$ (endemic means that the population contains a positive proportion of infected individuals, i.e. $z_{1}^{*}>0$ ), and a disease free equilibrium $\bar{z}$ (disease free means that in that equilibrium there is no infected individual, $\bar{z}_{1}=0$ ), which is unstable for the same range of values of the parameters, and $\widetilde{\partial O}=\left\{z, z_{1}=0\right\}$. In those two examples, we do not need to use the reflected solution of the SDE which will be defined below, since the reflected and unreflected processes are identical.

In the two other examples considered in Sections 6.4 and 6.5, the situation is more complex, both the endemic equilibrium $z^{*}$ and the disease-free equilibrium are locally stable equilibria of (1.3), in the sense that both have a basin or attraction, which is a given neighborhood in $A$ of the corresponding equilibrium. Those two basins of attraction are separated by a manifold of dimension $d-1$, which coincides with $\widetilde{\partial O}$, and contains a second endemic equilibrium, which is unstable, and is $\bar{z}$. More precisely, $\bar{z}$ is a hyperbolic point, which attracts all points of $\widetilde{\partial O}$, but starting from any point in a neighborhood of $\widetilde{\partial O}$ which is not in $\widetilde{\partial O}$, the solution of (1.3) is repelled by $\widetilde{\partial O}$, and is attracted by one of the two stable equilibria.

The paper is organized as follows. Section 2 is devoted to definitions and the statement of our assumptions. In Section 3, we define our reflecting Poissonian SDE and we formulate a large deviations principle satisfied by the latter. Section 4 presents some preliminary lemmas about the rate function. These lemmas are mostly adapted versions of those in chapter 6 of Freidlin and Wentzell [7] and in Day [3]. Section 5 discusses the large deviations of the exit measure. Finally, in Section 6 we prove that in four selected examples of infectious disease models, the quasi-potential has a unique minimum on $\widetilde{\partial O}$, hence for large $N$ the exit takes place close to that specific point with probability close to 1 .

\section{Notation And The MAin ASSUmption}

We define the following cone generated by the family of vectors $\left(h_{j}\right)_{j=1, \ldots, k}$

$$
\mathcal{C}=\left\{y \in \mathbb{R}^{d}: y=\sum_{j=1}^{k} \mu^{j} h_{j}, \mu^{j} \geq 0 \quad \forall j\right\} .
$$

\footnotetext{
${ }^{1}$ In the example treated in Section 6.3, the notion of proportion has to be interpreted in an extended sense.
} 
We remark that in all the epidemics models that we will consider, the family of vectors $\left(h_{j}\right)_{j=1, \ldots, k}$ is such that $\mathcal{C}=\mathbb{R}^{d}$.

We now formulate some assumptions which are useful in order to obtain the large deviations principle of the reflected Poissonian SDE that we will construct in Section 3, and which we will assume to hold throughout this paper, without recalling them in the statements.

\section{Assumption 2.1.}

1. $\mathcal{C}=\mathbb{R}^{d}$.

2. There exists a point $z_{0}$ in the interior of $O$ such that each segment joining $z_{0}$ and any $z \in \partial O$ does not touch any other point of the boundary $\partial O$.

3. There exists a constant $c$ such that for each $a>0$ small enough, $z \in \bar{O}$, if we denote $z^{a}=z+a\left(z_{0}-z\right)$,

$$
\operatorname{dist}\left(z^{a}, \partial O\right) \geq c a
$$

We shall assume that $c$ is choosen such that $\left|z-z^{a}\right| \leq c^{-1} a$ for all $z \in \bar{O}, a>0$.

4. The rate functions $\beta_{j}$ are Lipschitz continuous with the Lipschitz constant less than or equal to $C$.

5. There exist two constants $\lambda_{1}$ and $\lambda_{2}$ such that whenever $z \in \bar{O}$ is such that $\beta_{j}(z)<\lambda_{1}, \beta_{j}\left(z^{a}\right)>\beta_{j}(z)$ for all $a \in] 0, \lambda_{2}\left[^{2}\right.$.

6. There exist $\nu \in] 0,1 / 2\left[\right.$ and $a_{0}>0$ such that $C_{a} \geq \exp \left\{-a^{-\nu}\right\}$ for all $0<a<a_{0}$, where

$$
C_{a}=\inf _{j} \inf _{z: \operatorname{dist}(z, \partial O) \geq a} \beta_{j}(z) .
$$

\section{Reflected solution of a Poisson dRiven SDE, and large DEVIATIONS PRINCIPLE}

In this section, we define a notion of Poisson driven SDE "reflected at the boundary of $O$ ", and recall the large deviations principle for its solution, which has been established in [12]. For any $z \in \bar{O}$, let

$$
z^{N}=\left\{\begin{array}{lc}
\frac{[N z]}{N} & \text { if } \frac{[N z]}{N} \in \bar{O} \\
\underset{y \in \bar{O}^{(N)}}{\arg \inf }|y-z| & \text { otherwise }
\end{array}\right.
$$

and $\tilde{Z}_{t}^{N}$ denote the d-dimensional processes defined by

$$
\begin{aligned}
\tilde{Z}^{N}(t)=\tilde{Z}^{N, z}(t) & :=z^{N}+\frac{1}{N} \sum_{j=1}^{k} h_{j} Q_{t}^{N, j}-\frac{1}{N} \sum_{j=1}^{k} h_{j} \int_{0}^{t} \mathbf{1}_{\left\{\tilde{Z}^{N, z}(s-)+\frac{h_{j}}{N} \notin \bar{O}\right\}} \mathrm{d} Q_{s}^{N, j} \\
& :=z^{N}+\frac{1}{N} \sum_{j=1}^{k} h_{j} \int_{0}^{t} \mathbf{1}_{\left\{\tilde{Z}^{N, z}(s-)+\frac{h_{j}}{N} \in \bar{O}\right\}} \mathrm{d} Q_{s}^{N, j},
\end{aligned}
$$

where for $j=1, \ldots, k, Q^{N, j}$ is given as

$$
Q_{t}^{N, j}=P_{j}\left(N \int_{0}^{t} \beta_{j}\left(\tilde{Z}^{N, z}(s)\right) \mathrm{d} s\right) .
$$

We then obtain a Poisson driven SDE whose solution takes its values in $O^{(N)}=A^{(N)} \cap \bar{O}$.

\footnotetext{
${ }^{2}$ This means that the $\beta_{j}$ 's are positive for any $z \in O$, and whenever $\beta_{j}(z)=0$ for $z$ belonging to some part of $\partial O, \beta_{j}$ is strictly increasing along a trajectory which moves away from that part of the boundary.
} 
Note that our reflection affects only the behaviour of $Z_{t}^{N}$ near the part $\widetilde{\partial O}$ of the boundary of $O$. Note also that the rate function of the large deviations of the solutions of the reflected and unreflected SDEs coincide. This is reminiscent of the situation for Brownian driven SDEs, where the same is true, provided the reflection is "co-normal", see e.g. $[3,5]$.

Let $D_{T, \bar{O}}$ denote the set of functions from $[0, T]$ into $\bar{O}$ which are right continuous and have left limits, $\mathcal{A C}_{T, \bar{O}} \subset D_{T, \bar{O}}$ the subspace of absolutely continuous functions. For any $\phi, \psi \in D_{T, \bar{O}}$ and $W$ a subset of $D_{T, \bar{O}}$ let

$$
\|\phi-\psi\|_{T}=\sup _{t \leq T}\left|\phi_{t}-\psi_{t}\right|
$$

where |.| denotes Euclidian distance in $\mathbb{R}^{d}$ and

$$
\rho_{T}(\phi, W)=\inf _{\varphi \in W}\|\phi-\varphi\|_{T}
$$

For all $\phi \in \mathcal{A C}_{T, \bar{O}}$, let $\mathcal{A}_{d}(\phi)$ denote the (possibly empty) set of vector-valued Borel measurable functions $\mu$ such that for all $j=1, \ldots, k, 0 \leq t \leq T, \mu_{t}^{j} \geq 0$ and

$$
\frac{\mathrm{d} \phi_{t}}{\mathrm{~d} t}=\sum_{j=1}^{k} \mu_{t}^{j} h_{j}, \quad \mathrm{t} \text { a.e. }
$$

We define the rate function

$$
I_{T}(\phi):= \begin{cases}\inf _{\mu \in \mathcal{A}_{d}(\phi)} I_{T}(\phi \mid \mu), & \text { if } \phi \in \mathcal{A C}_{T, \bar{O}} \\ \infty, & \text { else }\end{cases}
$$

where

$$
I_{T}(\phi \mid \mu)=\int_{0}^{T} \sum_{j=1}^{k} f\left(\mu_{t}^{j}, \beta_{j}\left(\phi_{t}\right)\right) \mathrm{d} t
$$

with $f(\nu, \omega)=\nu \log (\nu / \omega)-\nu+\omega$. We assume in the definition of $f(\nu, \omega)$ that for all $\nu>0, \log (\nu / 0)=\infty$ and $0 \log (0 / 0)=0 \log (0)=0$. By the definition of $f$, it is not difficult to remark that

$$
I_{T}(\phi)=0 \text { if and only if } \phi \text { solves the } O D E(1.3) .
$$

The above rate function can also be defined as

$$
I_{T}(\phi):= \begin{cases}\int_{0}^{T} L\left(\phi_{t}, \phi_{t}^{\prime}\right) \mathrm{d} t & \text { if } \phi \in \mathcal{A C}_{T, \bar{O}} \\ \infty & \text { else. }\end{cases}
$$

where for all $z \in \bar{O}, y \in \mathbb{R}^{d}$

$$
L(z, y)=\sup _{\theta \in \mathbb{R}^{d}} \ell(z, y, \theta)
$$


with for all $z \in \bar{O}, y \in \mathbb{R}^{d}$ and $\theta \in \mathbb{R}^{d}$

$$
\ell(z, y, \theta)=\langle\theta, y\rangle-\sum_{j=1}^{k} \beta_{j}(z)\left(e^{\left\langle\theta, h_{j}\right\rangle}-1\right)
$$

The rate function defined above is a good rate function (cf. [8]), that is for all $s>0$, the set $\left\{\phi \in D_{T, \bar{O}}\right.$ : $\left.I_{T}(\phi) \leq s\right\}$ is compact. We now formulate a new assumption, and the result concerning the $L D P$ for our reflected model (3.2), which is proved in [12].

Assumption 3.1. There exists a function $u \in C_{b}^{1}(\bar{O})$ which satisfies the following assumptions:

1. $O=A \cap\{z \in \bar{O} ; u(z)>0\}, \widetilde{\partial O}=A \cap\{z \in \bar{O} ; u(z)=0\}$.

2. $\nabla u(z) \neq 0$, for all $z \in \widetilde{\partial O}$.

3. There exists $C_{1}, C_{2}>0$ such that $\min \left\{C_{1} \operatorname{dist}(z, \widetilde{\partial O}), C_{2}\right\} \leq u(z)$, for all $z \in \bar{O}$.

4. $\langle b(z), \nabla u(z)\rangle \geq 0$ for all $z \in \bar{O}$, with again $b(z)=\sum_{j=1}^{k} \beta_{j}(z) h_{j}$.

5. There exists $\rho>0$ such that $\left\langle-g_{N}(z), \nabla u(z)\right\rangle \leq \rho \sum_{j=1}^{k} \mathbf{1}_{\left\{z+\frac{h_{j}}{N} \notin \bar{O}\right\}}$, where

$$
g_{N}(z)=\sum_{j=1}^{k} \mathbf{1}_{\left\{z+\frac{h_{j}}{N} \notin \bar{O}\right\}} \beta_{j}(z) h_{j} .
$$

Note that in the examples treated in Sections 6.2 and 6.3, assumption 3.1 is satisfied with $u(z)=z_{1}$, while this assumption holds true in the two examples treated in Sections 6.4 and 6.5, as explained in section 7 of [12].

Theorem 3.2. Let $\left\{\tilde{Z}_{t}^{N, z}, 0 \leq t \leq T\right\}$ be the solution of (3.2).

a) For $z \in \bar{O}, \phi \in D_{T, \bar{O}}, \phi_{0}=z, \eta>0$ and $\delta>0$ there exists $N_{\eta, \delta} \in \mathbb{N}$ such that for all $N>N_{\eta, \delta}$

$$
\mathbb{P}_{z}\left(\left\|\tilde{Z}^{N}-\phi\right\|_{T}<\delta\right) \geq \exp \left\{-N\left(I_{T}(\phi)+\eta\right)\right\}
$$

b) For any open subset $G$ of $D_{T, \bar{O}}$, the following holds uniformly over $z \in \bar{O}$

$$
\liminf _{N \rightarrow \infty} \frac{1}{N} \log \mathbb{P}_{z}\left(\tilde{Z}^{N} \in G\right) \geq-\inf _{\phi \in G, \phi_{0}=z} I_{T}(\phi) .
$$

c) For $z \in \bar{O}, \delta>0$ let

$$
H_{\delta}(s)=\left\{\phi \in D_{T, \bar{O}}:, \phi_{0}=z, \rho_{T}(\phi, \Phi(s)) \geq \delta\right\} \quad \text { where } \quad \Phi(s)=\left\{\psi \in D_{T, \bar{O}}: I_{T}(\psi) \leq s\right\}
$$

For any $\delta, \eta, s>0$ there exists $N_{0} \in \mathbb{N}$ such that for all $N>N_{0}$

$$
\mathbb{P}_{z}\left(\tilde{Z}^{N} \in H_{\delta}(s)\right) \leq \exp \{-N(s-\eta)\}
$$

d) For any closed subset $F$ of $D_{T, \bar{O}}$, the following holds uniformly over $z \in \bar{O}$

$$
\limsup _{N \rightarrow \infty} \frac{1}{N} \log \mathbb{P}_{z}\left(\tilde{Z}^{N} \in F\right) \leq-\inf _{\phi \in F, \phi_{0}=z} I_{T}(\phi) .
$$




\section{Notations AND ImPORTANT LEMMAS}

We assume from now on that there exists a (unique) point $z^{*} \in O$ such that for any $z \in O, Y(t, z) \rightarrow z^{*}$, as $t \rightarrow \infty$.

For $z, y \in \bar{O}$, we define the following functionals.

$$
\begin{aligned}
V_{\bar{O}}(z, y, T) & :=\inf _{\phi \in D_{T, \bar{O}}, \phi_{0}=z, \phi_{T}=y} I_{T}(\phi) \\
V_{\bar{O}}(z, y) & :=\inf _{T>0} V_{\bar{O}}(z, y, T) \\
V_{\widetilde{\partial O}} & :=\inf _{y \in \widetilde{\partial O}} V_{\bar{O}}\left(z^{*}, y\right) .
\end{aligned}
$$

We will denote by $B_{r}(y)$ the open ball centered at $y$ with radius $r$, and $B_{r}(K)=\cup_{y \in K} B_{r}(y)$. For large $N$, the function $V_{\bar{O}}(z, y)$ quantifies the energy needed for a trajectory to deviate from being a solution of the ODE (1.3), and go from $z$ to $y$, without leaving $\bar{O}=O \cup \widetilde{\partial O}$ and $V_{\widetilde{\partial O}}$ is the minimal energy required to hit the boundary $\widetilde{\partial O}$ when starting from $z^{*}$. We now prove a few Lemmas, which are analogues of some Lemmas of chapter 6 in [7].

Lemma 4.1. There exists a constant $C>0$ and a function $\mathcal{K} \in C\left(\mathbb{R}_{+}, \mathbb{R}_{+}\right)$with $\mathcal{K}(0)=0$ such that for all $\rho>0$

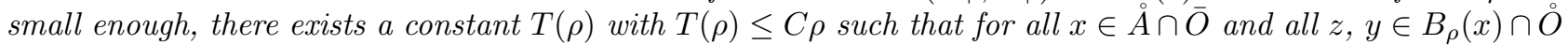
there exists an curve $\left(\phi_{t}\right)=\left(\phi_{t}(\rho, z, y)\right) 0 \leq t \leq T(\rho)$ with $\phi_{0}=z, \phi_{T(\rho)}=y$ entirely in $B_{\rho}(x) \cap \bar{O}$, such that $I_{T(\rho)}(\phi) \leq \mathcal{K}(T(\rho))$.

Proof. We will exploit Assumptions 2.1.3 and 2.1.6 and refer to the notations there. Note that the distance between $y$ and $z$ is at most $2 \rho$. Let $y^{a}$ and $z^{a}$ be the points defined in Assumption 2.1.3, with $a=2 \rho / c$. Then both are at distance at least $2 \rho$ from the boundary of $O$, while they are at distance less than $2 \rho$ one from another. Consequently the segment joining those two points is at distance at least $\sqrt{3} \rho$ from the boundary. We choose as function $\phi$ the piecewise linear function which moves at speed one, first in straight line from $z$ to $z^{a}$, then from $z^{a}$ to $y^{a}$, and finally from $y^{a}$ to $y$. The time needed to do so is bounded from above by $2\left(1+c^{-2}\right) \rho$. Thanks to Assumption 2.1.1, $I_{T(\rho)}(\phi)<\infty$. Refering to the formula (3.5) for the rate function and to Assumption 2.1.6, we see that the contribution of the straight line between $z^{a}$ and $y^{a}$ to $I_{T(\rho)}(\phi)$ is bounded by $C \rho^{1-\nu}$, while the contribution of the two other pieces is bounded by a universal constant times (below $\bar{c}=2 c^{-2}$ )

$$
\int_{0}^{\bar{c} \rho} \frac{\mathrm{d} t}{t^{\nu}}=\frac{\bar{c}^{1-\nu}}{1-\nu} \rho^{1-\nu}
$$

The result follows.

Lemma 4.2. $\forall \eta>0, K \subseteq O \bigcirc \widetilde{\partial O}$ compact, there exist $T_{0}$ such that for any $z, y \in K$ there exists a function $\phi_{t}, 0 \leq t \leq T$ satisfying $\phi_{0}=z, \phi_{T}=y, T \leq T_{0}$ such that $I_{T}(\phi) \leq V_{\bar{O}}(z, y)+\eta$.

Proof. As $K$ is compact there exists a finite number $M$ of points $\left\{z_{i}, 1 \leq i \leq M\right\}$ in $K$ such that

$$
K \subseteq \bigcup_{i=1}^{M} B_{r}\left(z_{i}\right)
$$

For all $z, y \in K$, there exist $1 \leq i, j \leq M$ with $z \in B_{r}\left(z_{i}\right), y \in B_{r}\left(z_{j}\right)$. Since $V_{\bar{O}}(u, v)$ is continuous, chosing $r$ small enough, we deduce that

$$
V_{\bar{O}}\left(z_{i}, z_{j}\right) \leq V_{\bar{O}}(z, y)+\frac{\eta}{8} .
$$


Moreover, from the finiteness of $V_{\bar{O}}$ we have that for all $z_{i}, z_{j}$, there exists $T^{i, j}$ and $\widetilde{\phi}_{t}$ with $\widetilde{\phi}_{0}=z_{i}, \widetilde{\phi}_{T^{i, j}}=z_{j}$

$$
I_{T^{i, j}}(\widetilde{\phi}) \leq V_{\bar{O}}\left(z_{i}, z_{j}\right)+\frac{\eta}{4}
$$

We can fix $T_{0}=\max _{i, j} T^{i, j}+2$ and Lemma 4.1 tells us that it is always possible to connect $z$ and $x_{i}$ resp $\left(z_{j}\right.$ and $y)$ with $\phi_{t}^{i}, 0 \leq t \leq T^{i}<1 \operatorname{resp}\left(\phi_{t}^{j}, 0 \leq t \leq T^{j}<1\right)$ such that $\phi_{0}^{i}=z, \phi_{T^{i}}^{i}=z_{i} \operatorname{resp}\left(\phi_{0}^{j}=z_{j}, \phi_{T^{j}}^{j}=y\right)$ and

$$
I_{T^{i}}\left(\phi^{i}\right) \leq \frac{\eta}{4} \operatorname{resp}\left(I_{T^{j}}\left(\phi^{j}\right) \leq \frac{\eta}{4}\right)
$$

Concatenating $\phi^{i}, \widetilde{\phi}$ and $\phi^{j}$, we obtain a trajectory $\phi$ with all the required properties.

Now, we define the equivalence relation " $\mathcal{R}$ " in $\bar{O}$ by

$$
z \mathcal{R} y \quad \text { iff } \quad V_{\bar{O}}(z, y)=V_{\bar{O}}(y, z)=0
$$

Lemma 4.3. Suppose there exists $y \neq z$ such that $z \mathcal{R} y, y, z \in \bar{O}$. Then the trajectory $Y(t, z)$ of the dynamical system (1.3) starting from $z$ lies in the set of points $\{v \in \bar{O}: v \mathcal{R} z\}$.

Proof. As $z \mathcal{R} y$ there exists a sequence of functions $\phi_{t}^{n}, 0 \leq t \leq T_{n}, \phi_{0}^{n}=z, \phi_{T_{n}}=y$, with values in $\bar{O}$ and such that $I_{T_{n}}\left(\phi^{n}\right) \rightarrow 0$. The $T_{n}$ are bounded from below by a positive constant. Indeed there exists $n_{0} \in \mathbb{N}$ such that for all $n \geq n_{0}$

$$
I_{T_{n}}\left(\phi^{n} \mid \mu^{n}\right) \leq 1
$$

Now either $T_{n} \geq \sigma^{-1}$, where $\sigma:=\sup _{1 \leq j \leq k, z \in A} \beta_{j}(z)$, or else from the Lemma 1 in [11], for all $1 \leq j \leq k$,

$$
\int_{0}^{T_{n}} \mu_{t}^{n, j} \mathrm{~d} t \leq \frac{2}{-\log \left(\sigma T_{n}\right)} .
$$

Moreover $\frac{\mathrm{d} \phi_{t}^{n}}{\mathrm{~d} t}=\sum_{j=1}^{k} \mu_{t}^{n, j} h_{j}$, hence

$$
\begin{aligned}
|z-y| & \leq\left|\int_{0}^{T_{n}} \frac{\mathrm{d} \phi_{t}^{n}}{\mathrm{~d} t} \mathrm{~d} t\right| \\
& \leq \sqrt{d} \sum_{j=1}^{k} \int_{0}^{T_{n}} \mu_{t}^{n, j} \mathrm{~d} t
\end{aligned}
$$

thus there exists $1 \leq j \leq k$ such that

$$
\frac{1}{k \sqrt{d}}|z-y| \leq \int_{0}^{T_{n}} \mu_{t}^{n, j} \mathrm{~d} t .
$$

Now, combining (4.2) and (4.3) we deduce that

$$
T_{n} \geq \sigma^{-1} \exp \left(-\frac{2 k \sqrt{d}}{|z-y|}\right)>0,
$$


which shows that $T_{n} \geq T$ for all $n$ and some $T>0$. Now $I_{T}\left(\phi_{t}^{n}\right)$ converges to 0 as $n \rightarrow \infty$. Therefore, for a constant $s>0$, there exists $n_{0} \in \mathbb{N}$ such that for all $n \geq n_{0}, I_{T}\left(\phi^{n}\right)<s$. By the compactness of the set $\left\{\psi: I_{T}(\psi) \leq s\right\}$, there exists a subsequence $\left(\phi^{n_{k}}\right)_{n_{k}}$ of these functions which converges, uniformly on $[0, T]$, to a function $\phi_{t}$. As $I_{T}$ is lower semicontinuous, we have

$$
0=\liminf _{k \rightarrow \infty} I_{T}\left(\phi^{n_{k}}\right) \geq I_{T}(\phi) .
$$

Thus $I_{T}(\phi)=0$ and $\phi$ is the trajectory of the dynamical system (1.3) starting from $z$. The points $\phi_{t}, 0 \leq t \leq T$, are equivalent to $z$ and $y$ since we have $V_{\bar{O}}\left(z, \phi_{t}^{n}\right)$ and $V_{\bar{O}}\left(\phi_{t}^{n}, y\right)$ do not exceed $I_{T_{n}}\left(\phi^{n}\right) \rightarrow 0$ as $n \rightarrow \infty$.

Let $u$ be one of the points $z$ and $y$ such that that $\left|\phi_{T}-u\right| \geq \frac{1}{2}|z-y|$ then $\phi_{T} \mathcal{R} u$. In the same way as earlier, we can find some time interval in which the points of the dynamical system starting from $\phi_{T}$ are equivalent to $u$. We obtain the result by a successive application of the above reasoning, as in the proof of Lemma 1.5 page 165 in [7].

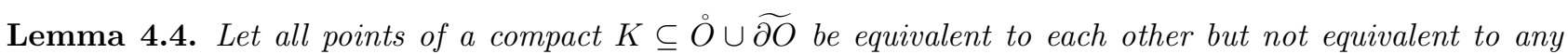
other point in $O \cup \widetilde{\partial O}$. For any $\eta>0, \delta>0$ and $z, y \in K$ there exists a function $\phi_{t}, 0 \leq t \leq T, \phi_{0}=z, \phi_{T}=y$, entirely in the intersection of $\bar{O}$ with the $\delta$-neighborhood of $K$ and such that $I_{T}(\phi)<\eta$.

Proof. As $z, y \in K$ there exists a sequence of functions $\phi_{t}^{n}, 0 \leq t \leq T_{n}, \phi_{0}^{n}=z, \phi_{T_{n}}^{n}=y$, with values in $O \cup \widetilde{\partial O}$ and such that $I_{T_{n}}\left(\phi^{n}\right) \rightarrow 0$. And then there exists $n_{0} \in \mathbb{N}$ such that for all $n>n_{0}, I_{T_{n}}\left(\phi^{n}\right)<\eta$. If all curves $\phi_{t}^{n}$ with $n>n_{0}$ left the $\delta$-neighborhood of $K$, then they would have a limit point $x$ outside of this $\delta$-neighborhood and we have $V_{\bar{O}}(z, y)=V_{\bar{O}}(y, z)=0$ thus $x$ is equivalent to $z$ and $y$. A contradiction since all points of a compact $K$ are equivalent to each other but not equivalent to any other point in $\bar{O}$.

Lemma 4.5. Let $K$ be a compact subset of $O \cup \widetilde{\partial O}$ not containing any $\omega$-limit set ${ }^{3}$ of the dynamical system (1.3) entirely. There exist two positive constants $C$ and $T_{0}$ such that for all sufficiently large $N$, any $T>T_{0}$ and $z \in K$ we have

$$
\mathbb{P}_{z}\left(\tau_{K}^{N}>T\right) \leq \exp \left\{-N C\left(T-T_{0}\right)\right\}
$$

where $\tau_{K}^{N}$ is the time of first exit of $\tilde{Z}_{t}^{N}$ from $K$ and under $\mathbb{P}_{z}, \tilde{Z}_{t}^{N}$ starts from the point $z^{N}$ defined by (3.1).

Proof. As $K$ does not contain any $\omega$-limit set entirely, we can choose $\delta$ sufficiently small such that the closed $\delta$-neighborhood $K^{\delta}$ does not contain any $\omega$-limit set entirely, either. For $z \in K^{\delta}$, let

$$
\tau(z)=\inf \left\{t>0: Y(t, z) \notin K^{\delta}\right\} .
$$

where $Y(t, z)$ is the solution of (1.3) starting from $z$. We have $\tau(z)<\infty$ for all $z \in K^{\delta}$. By the continuous dependence of a solution on the initial conditions, the function $\tau(z)$ is upper semi-continuous, and then it attains its largest value $T_{1}=\sup _{z \in K^{\delta}} \tau(z)<\infty$.

Fix $T_{0}=T_{1}+1$ and let $\mathcal{F}^{K^{\delta}}$ the set of all functions $\phi_{t}$ defined for $0 \leq t \leq T_{0}$ and assuming values only in $K^{\delta}$. the set of these functions is closed in the sense of uniform convergence and because $I_{T_{0}}$ is lower semi-continuous,

$$
I_{0}=\min _{\phi \in \mathcal{F}^{K}} I_{T_{0}}(\phi) \quad \text { is attained on } \mathcal{F}^{K^{\delta}} .
$$

Moreover for all $\phi \in \mathcal{F}^{K^{\delta}}, I_{T_{0}}(\phi)>I_{0}>0$ since there are no trajectories of the dynamical system (1.3) in $\mathcal{F}^{K^{\delta}}$. If a function $\phi$ spend a time $T$ in $K$ with $T$ longer than $T_{0}$ we have $I_{T}(\phi) \geq I_{0}$; for the functions $\phi$ spending

\footnotetext{
${ }^{3}$ Given a dynamical system $Y(t, z)$, a point $\bar{z}$ is called an $\omega$-limit point of $z_{0}$ if there exists a sequence $\left(t_{n}\right)_{n \geq 1}$ such that $t_{n} \rightarrow \infty$ as $n \rightarrow \infty$, and $Y\left(t_{n}, z_{0}\right) \rightarrow \bar{z}$ as $n \rightarrow \infty$. The set of all such points is called the $\omega$-limit set of $Y\left(t, z_{0}\right)$ and denoted $\omega\left(z_{0}\right)$.
} 
time $T \geq 2 T_{0}$ in $K$ we have $I_{T} \geq 2 I_{0}$, and so on. We deduce that for all $\phi$ spending time $T$ in $T>T_{0}$ in $K$ we have

$$
I_{T}(\phi) \geq I_{0}\left[\frac{T}{T_{0}}\right]>I_{0}\left(\frac{T}{T_{0}}-1\right)=\frac{I_{0}}{T_{0}}\left(T-T_{0}\right)
$$

For $z \in K$ the functions in the set

$$
\Phi_{z}\left(I_{0}\right)=\left\{\phi: \phi_{0}=z, I_{T_{0}}(\phi) \leq I_{0}\right\}
$$

leave $K^{\delta}$ during the time from 0 to $T_{0}$; the trajectories $\tilde{Z}_{t}^{N, z}$ for which $\tau_{K}^{N}>T_{0}$, are at a distance greater than $\delta$ to this set. We deduce by using Theorem 3.2 that for any $z \in K$

$$
\begin{aligned}
\mathbb{P}_{z}\left(\tau_{K}^{N}>T_{0}\right) & \leq \mathbb{P}\left(\rho_{T_{0}}\left(\tilde{Z}^{N}, \Phi_{z}\left(I_{0}\right)\right) \geq \delta\right) \\
& \leq \exp \left\{-N\left(I_{0}-\eta\right)\right\} .
\end{aligned}
$$

Now we use the Markov property and we have

$$
\begin{aligned}
\mathbb{P}_{z}\left(\tau_{K}^{N}>(n+1) T_{0}\right) & \leq \mathbb{E}_{z}\left(\tau_{K}^{N}>n T_{0} ; \mathbb{P}_{\tilde{Z}^{N}\left(n T_{0}\right)}\left(\tau_{K}^{N}>T_{0}\right)\right) \\
& \leq \mathbb{P}_{z}\left(\tau_{K}^{N}>n T_{0}\right) \sup _{y \in K} \mathbb{P}_{y}\left(\tau_{K}^{N}>T_{0}\right)
\end{aligned}
$$

We obtain by induction that

$$
\begin{aligned}
\mathbb{P}_{z}\left(\tau_{K}^{N}>T\right) & \leq \mathbb{P}_{z}\left(\tau_{K}^{N}>\left[\frac{T}{T_{0}}\right] T_{0}\right) \\
& \leq\left(\sup _{y \in K} \mathbb{P}_{y}\left(\tau_{K}^{N}>T_{0}\right)\right)^{\left[\frac{T}{T_{0}}\right]} \\
& \leq \exp \left\{-N\left[\frac{T}{T_{0}}\right]\left(I_{0}-\eta\right)\right\}
\end{aligned}
$$

Hence the result with $c=\frac{I_{0}-\eta}{T_{0}}$, where $\eta$ is an arbitrary small number.

The following assumption comes essentially from [7] (p. 150).

Assumption 4.6. There exists a finite number of compacts $K_{1}, \ldots, K_{M} \subseteq \widetilde{\partial O}$ such that

(1) $z, y \in K_{i}$ implies $z \mathcal{R} y$

(2) $z \in K_{i}, y \notin K_{i}$ implies $z \mathcal{R} y$

(3) every $\omega$-limit set of $z \in \widetilde{\partial O}$ associated to (1.3) is contained entirely in one of the $K_{i}$.

Remark 4.7. In the four examples presented in Section 6, Assumption 4.6 is satisfied with $M=1$ and $K_{1}=\{\bar{z}\}$, where $\bar{z}$ is the limit as $t \rightarrow \infty$ of $Y(t, z)$, for all $z \in \widetilde{\partial O}$.

We moreover define $K_{0}=\left\{z^{*}\right\}$. We now construct as in [3, 7] an embedded Markov chain $\tilde{Z}_{n}$ associated to the process $\tilde{Z}^{N}(t)$ in the following way: let $\rho_{0}$ and $\rho_{1}$ such that $0<\rho_{1}<\rho_{0}<\frac{1}{2} \min _{i \neq j} \operatorname{dist}\left(K_{i}, K_{j}\right)$,

$$
\begin{aligned}
G_{i}^{1} & :=\overline{B_{\rho_{1}}\left(K_{i}\right)} \\
C & :=\bar{O} \backslash \bigcup_{0}^{M} B_{\rho_{0}}\left(K_{i}\right) ;
\end{aligned}
$$




$$
\begin{aligned}
\Gamma_{i} & :=B_{2 \rho_{0}}\left(K_{i}\right) \backslash B_{\rho_{0}}\left(K_{i}\right) ; \\
\theta_{0} & :=0 \\
\sigma_{n} & :=\inf \left\{t \geq \theta_{n}: \tilde{Z}^{N}(t) \in C\right\} ; \\
\theta_{n+1} & :=\inf \left\{t \geq \sigma_{n}: \tilde{Z}^{N}(t) \in \bigcup_{0}^{M} G_{i}^{1}\right\} ; \\
\tilde{Z}_{n} & :=\tilde{Z}^{N}\left(\theta_{n}\right) .
\end{aligned}
$$

Define moreover

$$
\tau_{O}^{N}:=\inf \left\{t>0: \tilde{Z}^{N}\left(t^{-}\right)+\frac{1}{N} \sum_{j=1}^{k} h_{j} \Delta Q_{t}^{j} \notin \bar{O} \text { or } \tilde{Z}^{N}(t) \in \widetilde{\partial O}\right\}
$$

where $Q^{j}$ is defined by (3.3) and $\Delta Q_{t}^{j}=Q_{t}^{j}-Q_{t^{-}}^{j}$.

We also introduce the quantities $\tilde{V}_{\bar{O}}(z, y), \tilde{V}_{\bar{O}}\left(z, K_{i}\right), \tilde{V}_{\bar{O}}\left(K_{i}, K_{j}\right)$ and $V_{\bar{O}, K_{0}^{c}}(z, y)$ defined as in [7] by: $\forall z, y \in \bar{O}$

$$
\begin{aligned}
\widetilde{V}_{\bar{O}}(z, y): & =\inf \left\{I_{T}(\phi): T>0, \phi_{0}=z, \phi_{T}=y, \phi_{t} \in \bar{O} \backslash \bigcup_{\ell=0}^{M} K_{\ell} \forall t \in(0, T)\right\} \\
\widetilde{V}_{\bar{O}}\left(z, K_{i}\right): & =\inf \left\{I_{T}(\phi): T>0, \phi_{0}=z, \phi_{T} \in K_{i}, \phi_{t} \in \bar{O} \backslash \bigcup_{\ell \neq i} K_{\ell} \forall t \in(0, T)\right\} \\
\widetilde{V}_{\bar{O}}\left(K_{i}, K_{j}\right): & =\inf \left\{I_{T}(\phi): T>0, \phi_{0} \in K_{i}, \phi_{T} \in K_{j}, \phi_{t} \in \bar{O} \backslash \bigcup_{\ell \neq i, j} K_{\ell} \forall t \in(0, T)\right\} \\
V_{\bar{O}, K_{0}^{c}}(z, y): & =\inf \left\{I_{T}(\phi): T>0, \phi_{0}=z, \phi_{T}=y, \phi_{t} \in \bar{O} \backslash K_{0} \forall t \in(0, T)\right\} .
\end{aligned}
$$

We now establish the following equality (recall (4.11) and the definition of $V_{\widetilde{\partial O}}$ at the beginning of the present section).

Lemma 4.8. We have

$$
V_{\widetilde{\partial O}}:=\min _{i=1}^{M} \widetilde{V}_{\bar{O}}\left(K_{0}, K_{i}\right)
$$

Proof. We fix $\varepsilon>0$ arbitrary. Let $\phi_{t}, 0 \leq t \leq T$ be such that $\phi_{0}=z^{*}, \phi_{T} \in \widetilde{\partial O}$ and $I_{T}(\phi)<V_{\widetilde{\partial O}}+\varepsilon$. If $\phi_{t} \in \bigcup_{i=1}^{M} K_{i}$ for some $t \in[0, T)$, we can find $0 \leq t_{0}<t_{1}<T$ such that $\phi_{t_{0}}=z^{*}, \phi_{t} \notin \bigcup_{i=1}^{M} K_{i}$ for all $t_{0}<t<t_{1}$ and $\phi_{t_{1}} \in K_{j}$ for some $j$. Thus $\widetilde{V}_{\bar{O}}\left(K_{0}, K_{j}\right) \leq V_{\widetilde{\partial O}}+\varepsilon$. Otherwise if $\phi_{t}$ has reached $\widetilde{\partial O}$ but avoided $\bigcup_{i=1}^{M} K_{i}$, then we can extend $\phi_{t}$ to $t>T$ as a solution of (1.3). By the assumption $4.6(3), \phi_{t}$ comes arbitrarily close to $\bigcup_{1}^{M} K_{i}$ as $t \rightarrow+\infty$, but without any increase in the value of $I_{T}(\phi)$. It follows that

$$
\min _{i=1}^{M} \widetilde{V}_{\bar{O}}\left(K_{0}, K_{i}\right) \leq V_{\widetilde{\partial O}}+\varepsilon
$$

As $\varepsilon$ is arbitrary and the reverse inequality, $V_{\widetilde{\partial O}} \leq \min _{i=1}^{M} \widetilde{V}_{\bar{O}}\left(K_{0}, K_{i}\right)$, is obvious, we have the result. 
Lemma 4.9. For all $\eta>0$ there exists $\rho_{0}$ small enough such that for any $\rho_{2}, 0<\rho_{2}<\rho_{0}$, there exits $\rho_{1}$, $0<\rho_{1}<\rho_{2}$ such that for all $N$ large enough, for all $z$ in the $\rho_{2}$-neighborhood $G_{i}^{2}$ of the compact $K_{i}(0 \leq i \leq M)$, and all $j \geq 0$ we have the inequalities:

$$
\exp \left\{-N\left(\widetilde{V}_{\bar{O}}\left(K_{i}, K_{j}\right)+\eta\right)\right\} \leq \mathbb{P}_{z}\left\{\widetilde{Z}_{1} \in G_{j}^{1}\right\} \leq \exp \left\{-N\left(\widetilde{V}_{\bar{O}}\left(K_{i}, K_{j}\right)-\eta\right)\right\}
$$

Proof. By using Lemma 4.1 let $\rho>0$ such that $T(\rho)<\eta / 3 \mathcal{K}$. We choose $\rho_{0}>0$ smaller than $\rho / 3$ and $\frac{1}{3} \min _{i, j} \operatorname{dist}\left(K_{i}, K_{j}\right)$, and $\rho_{2} \in\left(0, \rho_{0}\right)$. For any two compacts $K_{i}, K_{j}, i \neq j$, for which $\widetilde{V}_{\bar{O}}\left(K_{i}, K_{j}\right)<\infty$, we choose a function $\phi_{t}^{i, j}, 0 \leq t \leq T_{i, j}$, such that $\phi_{0}^{i, j} \in K_{i}, \phi_{T_{i, j}}^{i, j} \in K_{j}, \phi_{t}^{i, j}$ does not touch $\bigcup_{\ell \neq i, j} K_{\ell}$ and for which

$$
I_{T_{i, j}}\left(\phi^{i, j}\right) \leq \widetilde{V}_{\bar{O}}\left(K_{i}, K_{j}\right)+\frac{\eta}{6}
$$

Let

$$
\rho_{3}=\frac{1}{2} \min \left\{\operatorname{dist}\left(\phi_{t}^{i, j}, \bigcup_{\ell \neq i, j} K_{\ell}\right): 0 \leq t \leq T_{i, j}, i, j=0, \ldots, M\right\}
$$

We now choose $0<\rho_{1}<\min \left(\rho / 2, \rho_{2}, \rho_{3}\right)$, and $\delta$ smaller than $\rho_{1}, \rho_{0}-\rho_{2}$. By Lemma 4.1, for any $z \in G_{i}^{2}$, let $\left(\psi_{t}^{i, 1}, 0 \leq t \leq \bar{T}_{i, 1}\right)$ with $\psi_{0}^{i, 1}=z, \psi_{\bar{T}_{i, 1}}^{i, 1}=z^{\prime} \in K_{i}$ such that $\psi^{i, 1}$ stays in $G_{i}^{2}$ and

$$
I_{\bar{T}_{i, 1}}\left(\psi^{i, 1}\right) \leq \frac{\eta}{6}
$$

We also have $\operatorname{dist}\left(\psi^{i, 1}, C\right) \geq \delta$ where $C$ is defined by (4.5). Moreover according to Lemma 4.4, there exists a curve $\left(\psi_{t}^{i, 2}\right)_{t}, 0 \leq t \leq \bar{T}_{i, 2}$ in $G_{i}^{2}$ with $\psi_{0}^{i, 2}=z^{\prime}, \psi_{\bar{T}_{i, 2}}^{i, 2}=\phi_{0}^{i, j} \in K_{i}$ such that

$$
I_{\bar{T}_{i, 2}}\left(\psi^{i, 2}\right) \leq \frac{\eta}{6}
$$

We combine these curves with the curve $\phi_{t}^{i, j}$ and we obtain a function $\phi_{t}, 0 \leq t \leq T=\bar{T}_{i, 1}+\bar{T}_{i, 2}+T_{i, j}\left(\phi_{t}\right.$ and $T$ depend on $z \in G_{i}^{2}$ and $j$ ) and $\phi_{0}=z, \phi_{T} \in K_{j}$ such that:

$$
I_{T}(\phi) \leq \widetilde{V}_{\bar{O}}\left(K_{i}, K_{j}\right)+\frac{\eta}{2}
$$

If $j=i$ we define $\phi_{t}, 0 \leq t \leq T$ such that $\phi_{0}=z \in G_{i}^{2}, \phi_{T}=z^{\prime \prime} \in K_{i}$ and $\operatorname{dist}\left(z^{\prime}, z^{\prime \prime}\right)=\operatorname{dist}\left(z^{\prime}, K_{i}\right)$ and we have

$$
I_{T}(\phi) \leq \frac{\eta}{2}=\widetilde{V}_{\bar{O}}\left(K_{i}, K_{i}\right)+\frac{\eta}{2}
$$

It is easy using Lemmas 4.1 and 4.2 to justify that the lengths of the intervals of definition of the functions $\phi_{t}$ constructed for all possible compacts $K_{i}, K_{j}$ and point $z \in G_{i}^{2}$ can be bounded from above by a constant $T_{0}<\infty$. The functions $\phi_{t}$ can be extended to the intervals from $T$ to $T_{0}$ to be a solution of (1.3) so that $I_{T}(\phi)=I_{T_{0}}(\phi)$.

Any trajectory $\tilde{Z}^{N, z}(t)$ such that $\left\|\tilde{Z}^{N, z}-\phi\right\|_{T_{0}} \leq \delta$ reaches the $\delta$-neighborhood of $K_{j}$ without getting closer than $2 \rho_{1}-\delta$ to any of the other compacts and then $\tilde{Z}_{1}=\tilde{Z}^{N, z}\left(\theta_{1}\right) \in G_{j}^{1}$. Thus using the large deviations 
Theorem 3.2 , we deduce that there exists $N_{0}$ depending only on $\eta, T_{0}$ and $\delta$ such that for all $N \geq N_{0}$ we have

$$
\begin{aligned}
\mathbb{P}_{z}\left\{\tilde{Z}_{1} \in G_{j}^{1}\right\} & \geq \mathbb{P}_{z}\left(\rho_{T}\left(\tilde{Z}^{N}, \phi\right)<\delta\right) \\
& \geq \exp \left\{-N\left(I_{T_{0}}(\phi)+\frac{\eta}{2}\right)\right\} \\
& >\exp \left\{-N\left(\widetilde{V}_{\bar{O}}\left(K_{i}, K_{j}\right)+\eta\right)\right\}
\end{aligned}
$$

And the left inequality of the Lemma follows.

Using the strong Markov property, it is sufficient to prove the right inequality for $z \in \Gamma_{i}$. With our choice of $\rho_{0}$ and $\delta$, for any curve $\phi_{t}, 0 \leq t \leq T$ beginning in a point of $\Gamma_{i}$, touching the $\delta$-neighborhood of $G_{j}^{1}$ and not touching the compacts $K_{\ell}, \ell \neq i, j$ we have

$$
I_{T}(\phi) \geq \widetilde{V}_{\bar{O}}\left(K_{i}, K_{j}\right)-\eta / 2
$$

Using Lemma 4.5, there exists two constants $c$ and $T_{1}$ such that for all $N$ large enough and $z \in(O \cup \widetilde{\partial O}) \backslash G$ where $G=\bigcup_{i=1}^{M} G_{i}^{1}$, we have:

$$
\mathbb{P}_{z}\left(\theta_{1}>T\right) \leq \exp \left\{-N c\left(T-T_{1}\right)\right\} \quad \text { for all } T>T_{1}
$$

Now we fix a $T>T_{1}$ then any trajectory of $\tilde{Z}^{N, z}(t)$ beginning at a point $z \in \Gamma_{i}$ and being in $G_{j}^{1}$ at time $\theta_{1}$ and not touching the compacts $K_{\ell}, \ell \neq i, j$ either spends time $T$ without touching $G \cup \widetilde{\partial O}$ (i.e the event $\left\{\theta_{1}>T\right\}$ is realized) or reaches $G_{j}^{1}$ before time $T$ and in this second case, with the notation $\Phi_{z}(s)=\left\{\psi \in D_{T, \bar{O}}: \psi(0)=\right.$ $\left.z, I_{T}(\psi) \leq s\right\}$, the event

$$
\left\{\rho_{T}\left(\tilde{Z}^{N, z}, \Phi_{z}\left(\widetilde{V}_{\bar{O}}\left(K_{i}, K_{j}\right)-\eta / 2\right)\right) \geq \delta\right\} \text { is realized. }
$$

Hence, for any $z \in \Gamma_{i}$ we have from Lemma 4.5 and Theorem 3.2 (c) that for $N$ large enough

$$
\begin{aligned}
\mathbb{P}_{z}\left(\tilde{Z}^{N}\left(\theta_{1}\right) \in G_{j}^{1}\right) & \leq \mathbb{P}_{z}\left(\theta_{1}>T\right)+\mathbb{P}_{z}\left(\rho_{T}\left(\tilde{Z}^{N}, \Phi_{z}\left(\widetilde{V}_{\bar{O}}\left(K_{i}, K_{j}\right)-\eta / 2\right)\right) \geq \delta\right) \\
& \leq \exp \left\{-N c\left(T-T_{1}\right)\right\}+\exp \left\{-N\left(\widetilde{V}_{\bar{O}}\left(K_{i}, K_{j}\right)-2 \eta / 3\right)\right\} \\
& \leq 2 \exp \left\{-N\left(\widetilde{V}_{\bar{O}}\left(K_{i}, K_{j}\right)-2 \eta / 3\right)\right\} \quad \text { with } T \text { large enough } \\
& \leq \exp \left\{-N\left(\widetilde{V}_{\bar{O}}\left(K_{i}, K_{j}\right)-\eta\right)\right\} \quad N \text { large enough such that } \frac{\ln (2)}{N}<\frac{\eta}{3}
\end{aligned}
$$

The lemma is proved.

Before we present others lemmas which will be useful, let us define the sequence $\kappa_{n} \in \mathbb{N}$ for which $\tilde{Z}_{\kappa_{n}}=$ $\tilde{Z}^{N, z}\left(\theta_{\kappa_{n}}\right) \in G_{0}^{1}$. Note that the $\kappa_{n}$ are $\bar{Z}_{n}$ stopping times and that the $\theta_{n}$ and $\theta_{\kappa_{n}}$ are $\mathcal{G}_{t}^{N, z}$ stopping times, where $\mathcal{G}_{t}^{N, z}=\sigma\left\{\tilde{Z}^{N, z}(s), 0 \leq s \leq t\right\}$.

Lemma 4.10. For all $z \in O$,

$$
\lim _{N \rightarrow \infty} \mathbb{P}_{z}\left(\tau_{O}^{N}>\theta_{\kappa_{1}}\right)=1
$$

Proof. We note that the statement is equivalent to $\lim _{N \rightarrow \infty} \mathbb{P}_{z}\left(\tilde{Z}^{N}\left(\theta_{\kappa_{1}} \wedge \tau_{O}^{N}\right) \in G_{0}^{1}\right)=1$, and we prove that

$$
\lim _{N \rightarrow \infty} \mathbb{P}_{z}\left(\tilde{Z}^{N}\left(\theta_{\kappa_{1}} \wedge \tau_{O}^{N}\right) \notin G_{0}^{1}\right)=0 .
$$


It is enough to take $z \in O \backslash G_{0}^{1}$. Indeed if $z \in G_{0}^{1}$ by the strong Markov property,

$$
\begin{aligned}
\mathbb{P}_{z}\left(\tilde{Z}^{N}\left(\theta_{\kappa_{1}} \wedge \tau_{O}^{N}\right) \notin G_{0}^{1}\right) & =\mathbb{E}_{z}\left(\mathbb{E}_{z}\left(\mathbf{1}_{\left\{\tilde{Z}^{N}\left(\theta_{\kappa_{1}} \wedge \tau_{O}^{N}\right) \notin G_{0}^{1}\right\}} \mid \mathcal{F}_{\sigma_{0}}\right)\right) \\
& =\mathbb{E}_{z}\left(\mathbb{P}_{\tilde{Z}^{N}\left(\sigma_{0}\right)}\left(\tilde{Z}^{N}\left(\theta_{\kappa_{1}} \wedge \tau_{O}^{N}\right) \notin G_{0}^{1}\right)\right) \\
& \leq \sup _{v \in \Gamma_{0}} \mathbb{P}_{v}\left(\tilde{Z}^{N}\left(\theta_{\kappa_{1}} \wedge \tau_{O}^{N}\right) \notin G_{0}^{1}\right)
\end{aligned}
$$

Let furthermore $T:=\inf \left\{t \geq 0 \mid Y(t, z) \in B_{\rho_{1} / 2}\left(z^{*}\right)\right\}$. Since $Y(\cdot, z)$ is continuous and never reaches $\widetilde{\partial O}$, we have $\inf _{t \geq 0} \operatorname{dist}(Y(t, z), \widetilde{\partial O})=: \delta>0$. Hence we have the following implication:

$$
\sup _{t \in[0, T]}\left|\tilde{Z}_{t}^{N, z}-Y(t, z)\right| \leq \frac{\delta}{2} \Rightarrow \tilde{Z}^{N, z}\left(\theta_{\kappa_{1}} \wedge \tau_{O}^{N}\right) \in G_{0}^{1}
$$

In other words,

$$
\mathbb{P}_{z}\left(\tilde{Z}^{N}\left(\theta_{\kappa_{1}} \wedge \tau_{O}^{N}\right) \notin G_{0}^{1}\right) \leq \mathbb{P}_{z}\left(\sup _{t \in[0, T]}\left|\tilde{Z}^{N, z}(t)-Y(t, z)\right|>\frac{\delta}{2}\right)
$$

The right hand side of Inequality (4.15) converges to zero as $N \rightarrow \infty$ by the weak law of large numbers established in [12].

In the following, we present some lemmas which are analogue to the lemmas of [3].

Lemma 4.11. Given $\eta>0$, there exists $\rho_{0}>0$ (which can be chosen arbitrarily small) such that for any $\rho_{2} \in\left(0, \rho_{0}\right)$, there exists $\rho_{1} \in\left(0, \rho_{2}\right)$ and $N$ large enough such that for all $z \in G_{0}^{1}$

$$
\exp \left(-N\left(V_{\widetilde{\partial O}}+\eta\right)\right) \leq \mathbb{P}_{z}\left(\tilde{Z}_{1} \in \bigcup_{i=1}^{M} G_{i}^{1}\right) \leq \exp \left(-N\left(V_{\widetilde{\partial O}}-\eta\right)\right)
$$

Proof. We have

$$
\mathbb{P}_{z}\left(\tilde{Z}_{1} \in \bigcup_{i=1}^{M} G_{i}^{1}\right)=\sum_{i=1}^{M} \mathbb{P}_{z}\left(\tilde{Z}_{1} \in G_{i}^{1}\right)
$$

then we deduce from Lemma 4.9 that $0<\rho_{1}<\rho_{2}<\rho_{0}$ can be chosen in such a way that $\forall x \in G_{0}^{1}$,

$$
\begin{aligned}
& \exp \{-N \eta / 2\} \sum_{i=1}^{M} \exp \left\{-N V_{\bar{O}}\left(K_{0}, K_{i}\right)\right\} \leq \mathbb{P}_{z}\left(\tilde{Z}_{1} \in \bigcup_{i=1}^{M} G_{i}^{1}\right), \\
& \mathbb{P}_{z}\left(\tilde{Z}_{1} \in \bigcup_{i=1}^{M} G_{i}^{1}\right) \leq \exp \{N \eta / 2\} \sum_{i=1}^{M} \exp \left\{-N V_{\bar{O}}\left(K_{0}, K_{i}\right)\right\} .
\end{aligned}
$$

Moreover it is easy to see that

$$
\exp \left\{-N \min _{i} V_{\bar{O}}\left(K_{0}, K_{i}\right)\right\} \leq \sum_{i=1}^{M} \exp \left\{-N V_{\bar{O}}\left(K_{0}, K_{i}\right)\right\} \leq M \exp \left\{-N \min _{i} V_{\bar{O}}\left(K_{0}, K_{i}\right)\right\}
$$


Hence $\forall x \in G_{0}^{1}$,

$$
\begin{aligned}
& \exp \left\{-N\left(\min _{i} V_{\bar{O}}\left(K_{0}, K_{i}\right)+\eta / 2\right)\right\} \leq \mathbb{P}_{z}\left(\tilde{Z}_{1} \in \bigcup_{i=1}^{M} G_{i}^{1}\right) \\
& \mathbb{P}_{z}\left(\tilde{Z}_{1} \in \bigcup_{i=1}^{M} G_{i}^{1}\right) \leq \exp \left\{-N\left(\min _{i} V_{\bar{O}}\left(K_{0}, K_{i}\right)-\eta / 2-\frac{1}{N} \log M\right)\right\} .
\end{aligned}
$$

The result now follows from Lemma 4.8 if we choose $N$ such that

$$
N>\frac{2|\log (M)|}{\eta} .
$$

In the following essential Lemma, we establish that an exit through the characteristic boundary $\widetilde{\partial O}$ is relatively likely once $\tilde{Z}^{N, z}(t)$ is close to it.

For its proof, we shall need the following assumption

Assumption 4.12. We assume that our system satisfies one of the two following conditions.

A $\widetilde{\partial O}=\left\{z, z_{1}=0\right\}$ and for each $N \geq 1$ the first coordinate $Z_{1}^{N}(t)$ of the process $Z^{N}(t)$ takes the form

$$
Z_{1}^{N}(t)=z_{1}+\frac{1}{N} P_{1}\left(N \int_{0}^{t} \varphi_{r} Z_{1}^{N}(r) \mathrm{d} r\right)-\frac{1}{N} P_{2}\left(N \int_{0}^{t} \psi_{r} Z_{1}^{N}(r) \mathrm{d} r\right)
$$

where $P_{1}$ and $P_{2}$ are two mutually independent standard Poisson processes, the two processes $\varphi$ and $\psi$ are adapted to the filtration $\mathcal{G}_{t}^{N}=\sigma\left\{Z_{s}^{N}, 0 \leq s \leq t\right\}$, and there exist two constants $a, b>0$ such that $0 \leq \varphi_{t} \leq a, b \leq \psi_{t}$ for all $t \geq 0$ a.s.

B For any $y \in \widetilde{\partial O}, \delta>0$, the set $B_{\delta}(y) \cap(\bar{O})^{c} \cap A$ has a non empty interior.

Remark 4.13. Note that the two examples presented below in Sections 6.2 and 6.3 satisfy condition $\mathbf{A}$ and not condition B, while the two examples presented in Sections 6.4 and 6.5 satisfy condition $\mathbf{B}$ and not condition $\mathbf{A}$.

Remark 4.14. In the situation covered by condition $\mathbf{A}$, the two processes $Z^{N}$ and $\tilde{Z}^{N}$ coincide, and the results from [12] are of no use, given the results from [11], while the results in [12] are useful for us in this paper for the situation covered by condition $\mathbf{B}$. Indeed, the process $Z^{N}$ cannot exit the set $A$. Under condition $\mathbf{B}$, only the piece $\widetilde{\partial O}$ of the boundary of $O$ can be crossed by the process $Z^{N}$, see Figures 1 and 2 in Sections 6.4 and 6.5 for an illustration. Under condition $\mathbf{A}$, the process $Z^{N}$ cannot cross the boundary.

In the proof of our next result, we shall need the following technical result.

Lemma 4.15. Let $\Xi$ be a Poisson r.v. with mean $\lambda$. For any $\mu>\lambda$,

$$
\mathbb{P}(\Xi>\mu) \leq \exp \left\{-\left(\mu \log \left(\frac{\mu}{\lambda}\right)-\mu+\lambda\right)\right\} .
$$

Proof. It follows from Chebychef's inequality that for any $\theta>0$,

$$
\mathbb{P}(\Xi>\mu)=\mathbb{P}\left(e^{\theta \Xi}>e^{\theta \mu}\right) \leq \exp \left\{-\theta \mu+\left(e^{\theta}-1\right) \mu\right\} .
$$

The result follows by choosing the optimal $\theta=\log (\mu / \lambda)$.

We can now state and prove the following crucial result. 
Lemma 4.16. Given $\eta>0$, there exist $0<\gamma<\eta, 0<\rho<\eta$, and $N_{0}$ large enough so that whenever $\operatorname{dist}(z, \widetilde{\partial O})<\rho / 3$ and $N>N_{0}$,

$$
\mathbb{P}_{z}\left(\tau_{O}^{N}<\gamma ; \sup _{0 \leq t \leq \gamma}\left|\tilde{Z}^{N}(t)-z\right|<\rho\right) \geq \exp (-N \eta)
$$

Proof. In this proof, we will use both processes $Z^{N}$ and $\tilde{Z}^{N}$.

Step 1. Proof under Assumption 4.12

A) Recall that here $\tilde{Z}^{N}=Z^{N}$. Let $\gamma_{1}>0$ and $0<\rho_{1}<\rho$ to be chosen below. Let $B\left(\gamma_{1}, \rho_{1}, \rho\right) \subset D_{\gamma}(O)$ be specified by the following requirements $: \phi:\left[0, \gamma_{1}\right] \rightarrow O$ belongs to $B\left(\gamma_{1}, \rho_{1}, \rho\right)$ iff $\phi_{1}(t) \leq \rho / 3$ for all $0 \leq t \leq \gamma_{1}$, and $\phi_{1}\left(\gamma_{1}\right)<\rho_{1}$. Since $Z^{N}$ satisfies the large deviations principle, it follows from Lemma 4.1 that for any $0<\rho_{1}<\rho, \rho$ small enough, we can choose $\gamma_{1}>0$ such that

$$
\mathbb{P}\left(Z^{N} \in B\left(\gamma_{1}, \rho_{1}, \rho\right)\right) \geq \exp (-N \eta / 3) .
$$

We now consider the process

$$
Z^{N, 2}(t)=Z^{N}\left(\gamma_{1}+t\right), \quad 0 \leq t \leq \gamma_{2}
$$

Thanks to Assumption $4.12 \mathbf{A}, Z_{1}^{N, 2}(t) \leq X^{N}(t)$, where

$$
X^{N}(t)=Z_{1}^{N}\left(\gamma_{1}\right)+N^{-1} \tilde{P}_{1}\left(N a \int_{0}^{t} X^{N}(s) \mathrm{d} s\right)-N^{-1} \tilde{P}_{2}\left(N b \int_{0}^{t} X^{N}(s) \mathrm{d} s\right),
$$

with

$$
\begin{aligned}
& \left.\left.\tilde{P}_{1}(t)=P_{1}\left(N \int_{0}^{\gamma_{1}} \varphi_{s} Z_{1}^{N}(s)\right) \mathrm{d} s+t\right)-P_{1}\left(N \int_{0}^{\gamma_{1}} \varphi_{s} Z_{1}^{N}(s)\right) \mathrm{d} s\right), \\
& \left.\left.\tilde{P}_{2}(t)=P_{2}\left(N \int_{0}^{\gamma_{1}} \psi_{s} Z_{1}^{N}(s)\right) \mathrm{d} s+t\right)-P_{2}\left(N \int_{0}^{\gamma_{1}} \psi_{s} Z_{1}^{N}(s)\right) \mathrm{d} s\right) .
\end{aligned}
$$

Indeed, while $X^{N}(t)=Z_{1}^{N, 2}(t)$ (which is the case for $t>0$ small enough), if the first jump is positive, it hits $X^{N}$ first, while if it is negative it hits $Z_{1}^{N, 2}$ first. So that when $X^{N}$ and $Z_{1}^{N, 2}$ start to disagree, they verify $X^{N}(t)>Z_{1}^{N, 2}(t)$. Since the two processes cannot cross, $Z_{1}^{N, 2}(t) \leq X^{N}(t)$ for all $t>0$ a.s. Let us denote by $\tau_{e x t}^{N}$ the time of extinction of $X^{N}$. From the above comparison, we see that or any $\gamma_{2}>0$,

$$
\tau_{O}^{N}-\gamma_{1} \leq \tau_{e x t}^{N}, \quad \text { hence }\left\{\tau_{O}^{N}-\gamma_{1}<\gamma_{2}\right\} \supset\left\{\tau_{e x t}^{N}<\gamma_{2}\right\} .
$$

Let $\gamma=\gamma_{1}+\gamma_{2}$. There exist mutually independent standard Poisson process $\tilde{P}_{j}, 1 \leq j \leq k$, which are independent of $\sigma\left\{Z_{t}^{N}, 0 \leq t \leq \gamma_{1}\right\}$, and positive constants $c_{j}$ and $d_{j}, 1 \leq j \leq k$, such that

$$
\begin{aligned}
\left\{\tau_{O}^{N}<\gamma\right\} & \cap\left\{\sup _{0 \leq t \leq \gamma}\left|\tilde{Z}^{N}(t)-z\right|<\rho\right\} \\
& \supset\left\{Z^{N} \in B\left(\gamma_{1}, \rho_{1}, \rho\right)\right\} \cap\left\{\tau_{O}^{N}-\gamma_{1}<\gamma_{2}\right\} \cap_{j=1}^{k}\left\{\tilde{P}\left(c_{j} \gamma_{2} N\right) \leq d_{j} \rho N\right\} \\
& \supset\left\{Z^{N} \in B\left(\gamma_{1}, \rho_{1}, \rho\right)\right\} \cap\left\{\tau_{e x t}^{N}<\gamma_{2}\right\} \cap_{j=1}^{k}\left\{\tilde{P}\left(c_{j} \gamma_{2} N\right) \leq d_{j} \rho N\right\} .
\end{aligned}
$$

Note that $N X^{N}$ is a continuous time birth and death branching process starting from $N z_{1}^{N}\left(\gamma_{1}\right)$, with birth rate $a$ and death rate $b$. We can and do assume that $a>b$ (which is the case in our applications covered by 
the situation A), i.e. $N X^{N}$ is supercritical. If we combine formula (1) page 107 of Athreya and Ney [1] and the formula for the birth and death case page 109, we see that

$$
\mathbb{P}\left(\tau_{e x t}^{N}<\gamma_{2} \mid Z^{N}\left(\gamma_{1}\right)\right)=\left(\frac{b-b e^{(b-a) \gamma_{2}}}{a-b e^{(b-a) \gamma_{2}}}\right)^{N Z_{1}^{N}\left(\gamma_{1}\right)} .
$$

Now

$$
\begin{aligned}
& \mathbb{P}\left(\tau_{O}^{N}<\gamma, \sup _{0 \leq t \leq \gamma}\left|\tilde{Z}^{N}(t)-z\right|<\rho\right) \\
& \geq \mathbb{P}\left(Z^{N} \in B\left(\gamma_{1}, \rho_{1}, \rho\right)\right) \mathbb{P}\left(\left\{\tau_{e x t}^{N}<\gamma_{2}\right\} \cap_{j=1}^{2}\left\{\tilde{P}\left(c_{j} \gamma_{2} N\right) \leq d_{j} \rho N\right\} \mid Z^{N} \in B\left(\gamma_{1}, \rho_{1}, \rho\right)\right) \\
& \quad \times \prod_{j=3}^{k} \mathbb{P}\left(\tilde{P}\left(c_{j} \gamma_{2} N\right) \leq d_{j} \rho N\right) .
\end{aligned}
$$

First we choose $\alpha<\min _{j=1}^{k} \frac{d_{j}}{c_{j}}$ and let $\gamma_{2}=\alpha \rho$. It follows from the law of large numbers that $\prod_{j=3}^{k} \mathbb{P}\left(\tilde{P}\left(c_{j} \gamma_{2} N\right) \leq d_{j} \rho N\right) \rightarrow 1$ as $\rightarrow \infty$. Hence for any $\eta>0$, there exists $N_{1} \geq 1$ such that for all $N \geq N_{1}$,

$$
\prod_{j=3}^{k} \mathbb{P}\left(\tilde{P}\left(c_{j} \gamma_{2} N\right) \leq d_{j} \rho N\right) \geq \exp (-N \eta / 3)
$$

We now treat the second factor on the right of (4.17)

$$
\begin{aligned}
& \mathbb{P}\left(\left\{\tau_{\text {ext }}^{N}<\gamma_{2}\right\} \cap_{j=1}^{2}\left\{\tilde{P}\left(c_{j} \gamma_{2} N\right) \leq d_{j} \rho N\right\} \mid Z_{1}^{N}\left(\gamma_{1}\right) \leq \rho_{1}\right) \\
& \quad \geq \mathbb{P}\left(\tau_{\text {ext }}^{N}<\gamma_{2} \mid Z_{1}^{N}\left(\gamma_{1}\right) \leq \rho_{1}\right)-\sum_{j=1}^{2} \mathbb{P}\left(\tilde{P}\left(c_{j} \gamma_{2} N\right)>d_{j} \rho N\right) \\
& \quad \geq\left(\frac{b-b e^{(b-a) \gamma_{2}}}{a-b e^{(b-a) \gamma_{2}}}\right)^{N \rho_{1}}-\sum_{j=1}^{2} \exp \left(-N \rho\left\{d_{j} \log \left(\frac{d_{j}}{c_{j} \alpha}\right)-d_{j}+c_{j} \alpha\right\}\right) \\
& \quad \geq \exp \left(-N \rho_{1} \log \left[A_{\gamma_{2}}\right]\right)-2 \exp (-N \rho \delta)
\end{aligned}
$$

where we have used Lemma 4.15 for the second inequality, and used the notations

$$
A_{\gamma_{2}}=\frac{a-b e^{(b-a) \gamma_{2}}}{b-b e^{(b-a) \gamma_{2}}}, \quad \text { and } \delta=\min _{j=1}^{2} d_{j} \log \left(\frac{d_{j}}{c_{j} \alpha}\right)-d_{j}+c_{j} \alpha>0
$$

We now choose $\rho=\eta /(3 \delta)$, hence $\gamma_{2}=(\alpha \eta) /(3 \rho)$. We finally choose $\rho_{1}=\left[6 \log \left(\frac{3 \rho}{\alpha \eta a}\right)\right]^{-1} \eta$, so that $\rho_{1} \log \left[A_{\gamma_{2}}\right]=\eta / 6$. Consequently, provided $N \geq(6 \log [3]) / \eta$,

$$
\mathbb{P}\left(\left\{\tau_{e x t}^{N}<\gamma_{2}\right\} \cap_{j=1}^{2}\left\{\tilde{P}\left(c_{j} \gamma_{2} N\right) \leq d_{j} \rho N\right\} \mid Z_{1}^{N}\left(\gamma_{1}\right) \leq \rho_{1}\right) \geq \exp (-N \eta / 3) .
$$

The result follows by combining (4.17), (4.16), (4.18) and (4.19). 
Step 2. Proof under Assumption 4.12

B) Let

$$
G=\left\{\phi \in D_{\gamma, A}: \operatorname{dist}\left(\phi_{0}, \widetilde{\partial O}\right)<\rho / 3, \sup _{0 \leq t \leq \gamma}\left|\phi_{t}-\phi_{0}\right|<\rho / 2 \quad \text { and } \quad \inf \left\{t: \phi_{t} \in \bar{O}^{c}\right\}<\gamma\right\}
$$

$G$ is open for the Skorohod topology and as $Z^{N, z}$ satisfies the large deviations principle, we deduce that for all $z \in O$ such that $\operatorname{dist}(z, \widetilde{\partial O})<\rho / 3$ we have for $N$ large enough

$$
\mathbb{P}_{z}\left(Z^{N} \in G\right) \geq \exp \left\{-N\left(\inf _{\phi \in G} I_{\gamma}(\phi)+\eta / 4\right)\right\}
$$

Moreover from Lemma 4.1 we can choose $\gamma, \rho<\eta \operatorname{such}$ that $\inf _{\phi \in G} I_{\gamma}(\phi)<\eta / 4$. Consequently

$$
\mathbb{P}_{z}\left(Z^{N} \in G\right) \geq \exp \{-N \eta / 2\}
$$

We also have for all $z \in O$ with $\operatorname{dist}(z, \widetilde{\partial O})<\rho / 3$.

$$
\begin{aligned}
& \mathbb{P}_{z}\left(\tau_{O}^{N}<\gamma, \sup _{0 \leq t \leq \gamma}\left|\tilde{Z}^{N}(t)-z\right|<\rho\right) \\
& \geq \mathbb{P}_{z}\left(\tau_{O}^{N}<\gamma, \sup _{\tau_{O}^{N} \leq t \leq \gamma}\left|\tilde{Z}^{N}(t)-z\right|<\rho, \sup _{0 \leq t<\tau_{O}^{N}}\left|\tilde{Z}^{N}(t)-z\right|<\rho / 2\right) \\
& =\mathbb{P}_{z}\left(\sup _{\tau_{O}^{N} \leq t \leq \gamma}\left|\tilde{Z}^{N}(t)-z\right|<\rho\left|\tau_{O}^{N}<\gamma, \sup _{0 \leq t<\tau_{O}^{N}}\right| \tilde{Z}^{N}(t)-z \mid<\rho / 2\right) \\
& \quad \times \mathbb{P}_{z}\left(\tau_{O}^{N}<\gamma, \sup _{0 \leq t<\tau_{O}^{N}}\left|\tilde{Z}^{N}(t)-z\right|<\rho / 2\right) .
\end{aligned}
$$

Moreover we have

$$
\begin{aligned}
\mathbb{P}_{z}\left(\tau_{O}^{N}<\gamma, \sup _{0 \leq t<\tau_{O}^{N}}\left|\tilde{Z}^{N}(t)-z\right|<\rho / 2\right) & =\mathbb{P}_{z}\left(\tau_{O}^{N}<\gamma, \sup _{0 \leq t<\tau_{O}^{N}}\left|Z^{N}(t)-z\right|<\rho / 2\right) \\
& \geq \mathbb{P}_{z}\left(Z^{N} \in G\right) \geq \exp \{-N \eta / 2\}
\end{aligned}
$$

Using the strong Markov property, we also have

$$
\begin{aligned}
& \mathbb{P}_{z}\left(\sup _{\tau_{O}^{N} \leq t \leq \gamma}\left|\tilde{Z}^{N}(t)-z\right|<\rho\left|\tau_{O}^{N}<\gamma, \sup _{0 \leq t<\tau_{O}^{N}}\right| \tilde{Z}^{N}(t)-z \mid<\rho / 2\right) \\
& =\mathbb{E}_{z}\left(\mathbb{P}_{\tilde{Z}^{N}\left(\tau_{O}^{N}\right)}\left(\sup _{\tau_{O}^{N} \leq t \leq \gamma}\left|\tilde{Z}^{N}(t)-z\right|<\rho\right)\left|\tau_{O}^{N}<\gamma, \sup _{0 \leq t<\tau_{O}^{N}}\right| \tilde{Z}^{N}(t)-z \mid<\rho / 2\right) \\
& \geq \inf _{y:|y-z|<\rho / 2} \mathbb{P}_{y}\left\{\sup _{0 \leq t \leq \gamma}\left|\tilde{Z}^{N}(t)-z\right|<\rho\right) \\
& \geq \inf _{y:|y-z|<\rho / 2} \mathbb{P}_{y}\left(\sup _{0 \leq t \leq \gamma}\left|\tilde{Z}^{N}(t)-y\right|<\rho / 2\right) \\
& \geq \exp \{-N \eta / 2\},
\end{aligned}
$$


where the last inequality follows from Theorem 3.2 and Lemma 4.1. Combining (4.20), (4.21) and (4.22) we have the result.

In the proofs of the next lemmas we denote

$$
O_{\varepsilon}:=\{z \in O: \operatorname{dist}(z, \widetilde{\partial O})>\varepsilon\}
$$

and we establish some inequalities involving $\theta_{\kappa_{1}}$.

Lemma 4.17. For all $\eta, \rho_{0}>0, y \in \widetilde{\partial O}$, and $z \in O \backslash \bigcup_{0}^{M} B_{\rho_{0}}\left(K_{i}\right)$, there exist $\delta_{0}, \rho_{2}$ and $N_{0}$ so that for all $\rho_{1}<\rho_{2}$, for all $\delta<\delta_{0}$ and $N>N_{0}$

$$
\mathbb{P}_{z}\left(\tau_{O}^{N}<\theta_{\kappa_{1}} ;\left|\tilde{Z}^{N}\left(\tau_{O}^{N}\right)-y\right|<\delta\right) \geq \exp \left(-N\left(V_{\bar{O}, K_{0}^{c}}(z, y)+\eta\right)\right)
$$

Proof. First, by definition of $V_{\bar{O}, K_{0}^{c}}(z, y)$, there exist $\bar{T}$ and a curve $\psi_{t}, 0 \leq t \leq \bar{T}$ with $\psi_{0}=z, \psi_{\bar{T}}=y$ and $\psi_{t} \in \bar{O} \backslash K_{0}$ for all $0 \leq t \leq \bar{T}$ such that

$$
I_{\bar{T}}(\psi) \leq V_{K_{0}^{c}}(z, y)+\frac{\eta}{12} .
$$

We can next choose $a$ such that $\psi_{t}^{a}, 0 \leq t \leq \bar{T}$ defined by

$$
\psi_{t}^{a}=(1-a) \psi_{t}+a z_{0}
$$

$\operatorname{satisfies} \operatorname{dist}\left(\psi_{t}^{a}, \widetilde{\partial O}\right) \geq c a$ and

$$
I_{\bar{T}}\left(\psi^{a}\right) \leq I_{\bar{T}}(\psi)+\frac{\eta}{12}
$$

According to Lemma 4.1, there exist two functions $\psi_{t}^{a, 1}, 0 \leq t \leq T^{a, 1}$ and $\psi_{t}^{a, 2}, 0 \leq t \leq T^{a, 2}$ such that $\psi_{0}^{a, 1}=z$, $\psi_{T^{a, 1}}^{a, 1}=\psi_{0}^{a}, \psi_{0}^{a, 2}=\psi_{\bar{T}}^{a}, \psi_{T^{a, 2}}^{a, 2}=y$ and

$$
I_{T^{a, 1}}\left(\psi^{a, 1}\right) \leq \frac{\eta}{12} \quad \text { and } \quad I_{T^{a, 2}}\left(\psi^{a, 2}\right) \leq \frac{\eta}{12} .
$$

Combining $\psi_{t}^{a, 1}, 0 \leq t \leq T^{a, 1}, \psi_{t-T^{a, 1}}^{a}, T^{a, 1} \leq t \leq \bar{T}+T^{a, 1}$ and $\psi_{t-\bar{T}-T^{a, 1}}^{a, 2}, \bar{T}+T^{a, 1} \leq t \leq T^{a, 2}+\bar{T}+T^{a, 1}$ we obtain a function $\varphi_{t}, 0 \leq t \leq T=\bar{T}+T^{a, 1}+T^{a, 2}$

$$
I_{T}(\varphi) \leq V_{K_{0}^{c}}(z, y)+\frac{\eta}{3}
$$

Let $\rho_{1}<\rho_{0}, \delta<\min \left\{\frac{1}{2} c a, \frac{\rho_{0}}{2}\right\}$ and $H \subseteq D_{T, \bar{O}}$ be the set of functions $\phi$ having the following properties:

- $\left|\phi_{T}-y\right|<\frac{1}{2} \delta$

- $\phi_{t}$ does not intersect $\left(\bar{O} \backslash O_{\frac{1}{4} \delta}\right) \backslash B_{\frac{1}{2} \delta}(y)$

- $\phi_{t}$ does not intersect $\overline{B_{\rho_{1}}\left(z^{*}\right)}$

$H$ is open and $\varphi \in H$ (if $\varphi$ intersects $\overline{B_{\rho_{1}}\left(z^{*}\right)}$ we use again Lemma 4.1 to modify $\varphi$ ). From theorem 3.2, for all $N$ large enough we deduce by using (4.23) that

$$
\begin{aligned}
\log \mathbb{P}_{z}\left(\tilde{Z}^{N} \in H\right) & \geq-N\left(\inf _{\psi \in H, \psi_{0}=z} I_{T}(\psi)+\frac{\eta}{3}\right) \\
& \geq-N\left(V_{K_{0}^{c}}(z, y)+\frac{2 \eta}{3}\right) .
\end{aligned}
$$


Moreover we remark that $\tilde{Z}^{N, z} \in H$ implies $\left|\tilde{Z}^{N, z}\left(\tau_{O}^{N} \wedge T\right)-y\right|<\frac{1}{2} \delta$ and $T<\theta_{\kappa_{1}}$. So

$$
\log \mathbb{P}_{z}\left(\left|\tilde{Z}^{N}\left(\tau_{O}^{N} \wedge T\right)-y\right|<\frac{1}{2} \delta, T<\theta_{\kappa_{1}}\right) \geq-N\left(V_{K_{0}^{c}}(z, y)+\frac{2 \eta}{3}\right)
$$

We also have

$$
\begin{aligned}
\Lambda= & \mathbb{P}_{z}\left(\left|\tilde{Z}^{N}\left(\tau_{O}^{N}\right)-y\right|<\delta, \theta_{\kappa_{1}}>\tau_{O}^{N}\right) \\
\geq & \mathbb{P}_{z}\left(\left|\tilde{Z}^{N}\left(\tau_{O}^{N} \wedge T\right)-y\right|<\frac{1}{2} \delta,\left|\tilde{Z}^{N}\left(\tau_{O}^{N}\right)-y\right|<\delta, T<\tau_{O}^{N}<\theta_{\kappa_{1}}\right) \\
& +\mathbb{P}_{z}\left(\left|\tilde{Z}^{N}\left(\tau_{O}^{N} \wedge T\right)-y\right|<\frac{1}{2} \delta,\left|\tilde{Z}^{N}\left(\tau_{O}^{N}\right)-y\right|<\delta, \tau_{O}^{N} \leq T<\theta_{\kappa_{1}}\right) \\
& +\mathbb{P}_{z}\left(\left|\tilde{Z}^{N}\left(\tau_{O}^{N} \wedge T\right)-y\right|<\frac{1}{2} \delta,\left|\tilde{Z}^{N}\left(\tau_{O}^{N}\right)-y\right|<\delta, \tau_{O}^{N}<\theta_{\kappa_{1}} \leq T\right) \\
\geq & \Lambda_{1}+\Lambda_{2}+\Lambda_{3} .
\end{aligned}
$$

Further

$$
\begin{aligned}
\Lambda_{1} & =\mathbb{E}_{z}\left(\mathbb{P}_{z}\left(\left|\tilde{Z}^{N}(T)-y\right|<\frac{1}{2} \delta, \tau_{O}^{N}<\theta_{\kappa_{1}}, T<\theta_{\kappa_{1}} \wedge \tau_{O}^{N},\left|\tilde{Z}^{N}\left(\tau_{O}^{N}\right)-y\right|<\delta \mid \mathcal{F}_{T}\right)\right) \\
& =\mathbb{E}_{z}\left(\left|\tilde{Z}^{N}(T)-y\right|<\frac{1}{2} \delta, T<\theta_{\kappa_{1}} \wedge \tau_{O}^{N}, \mathbb{P}_{\tilde{Z}^{N}(T)}\left(\tau_{O}^{N}<\theta_{\kappa_{1}},\left|\tilde{Z}^{N}\left(\tau_{O}^{N}\right)-y\right|<\delta\right)\right) .
\end{aligned}
$$

Furthermore for any $x$ such that $\operatorname{dist}(x, y)<\delta / 2$

$$
\mathbb{P}_{x}\left(\left|\tilde{Z}^{N}\left(\tau_{O}^{N}\right)-y\right|<\delta, \tau_{O}^{N}<\theta_{\kappa_{1}}\right) \geq \mathbb{P}_{x}\left(\tau_{O}^{N}<\gamma, \sup _{0 \leq t \leq \gamma}\left|\tilde{Z}^{N}(t)-y\right| \leq \delta\right)
$$

for all $\gamma>0$. In particular with $\gamma$ selected as in Lemma 4.16, we deduce that

$$
\Lambda_{1} \geq \mathbb{P}_{z}\left(\left|\tilde{Z}^{N}(T)-y\right|<\frac{1}{2} \delta, T<\theta_{\kappa_{1}} \wedge \tau_{O}^{N}\right) \exp (-N \eta / 3)
$$

We also have

$$
\begin{aligned}
\Lambda_{2} & =\mathbb{P}_{z}\left(\left|\tilde{Z}^{N}\left(\tau_{O}^{N}\right)-y\right|<\frac{1}{2} \delta, \tau_{O}^{N} \leq T<\theta_{\kappa_{1}}\right) \\
& \geq \mathbb{P}_{z}\left(\left|\tilde{Z}^{N}\left(\tau_{O}^{N}\right)-y\right|<\frac{1}{2} \delta, \tau_{O}^{N} \leq T<\theta_{\kappa_{1}}\right) \exp (-N \eta / 3),
\end{aligned}
$$

since

$$
\left\{\left|\tilde{Z}^{N, z}\left(\tau_{O}^{N}\right)-y\right|<\frac{1}{2} \delta, \tau_{O}^{N} \leq T<\theta_{\kappa_{1}}\right\} \subseteq\left\{\left|\tilde{Z}^{N, z}\left(\tau_{O}^{N}\right)-y\right|<\delta, \tau_{O}^{N}<\theta_{\kappa_{1}}\right\}
$$


and $\Lambda_{3}>0$. Thus

$$
\begin{aligned}
\Lambda \geq & \mathbb{P}_{z}\left(\left|\tilde{Z}^{N}\left(\tau_{O}^{N} \wedge T\right)-y\right|<\frac{1}{2} \delta, T<\theta_{\kappa_{1}} \wedge \tau_{O}^{N}\right) \exp (-N \eta / 3) \\
& +\mathbb{P}_{z}\left(\left|\tilde{Z}^{N}\left(\tau_{O}^{N} \wedge T\right)-y\right|<\frac{1}{2} \delta, \tau_{O}^{N} \leq T<\theta_{\kappa_{1}}\right) \exp (-N \eta / 3) \\
\geq & \mathbb{P}_{z}\left(\left|\tilde{Z}^{N}\left(\tau_{O}^{N} \wedge T\right)-y\right|<\frac{1}{2} \delta, T<\theta_{\kappa_{1}}\right) \exp (-N \eta / 3) .
\end{aligned}
$$

It follows from (4.24) and (4.25) that

$$
\log \mathbb{P}_{z}\left(\left|\tilde{Z}^{N}\left(\tau_{O}^{N}\right)-y\right|<\delta, \tau_{O}^{N}<\theta_{\kappa_{1}}\right) \geq-N\left(V_{K_{0}^{c}}(z, y)+\eta\right) .
$$

The lemma follows.

Lemma 4.18. For all $\eta>0, y \in \widetilde{\partial O}$, there exists $\delta_{0}>0$ and $\rho_{0}>0$ (which can be chosen arbitrarily small) such that for any $\rho_{1}, 0<\rho_{1}<\rho_{0}$, for any $\delta<\delta_{0}$ there exists $N_{0}$ so that for all $N>N_{0}$ and any $z \in G_{0}^{1}$,

$$
\mathbb{P}_{z}\left(\tau_{O}^{N}<\theta_{\kappa_{1}} ;\left|\tilde{Z}^{N}\left(\tau_{O}^{N}\right)-y\right|<\delta\right) \geq \exp \left(-N\left(V_{\bar{O}}\left(z^{*}, y\right)+\eta\right)\right) .
$$

Proof. Let $\rho_{1}<\rho_{0}, \forall z \in G_{0}^{1}$

$$
\begin{aligned}
\mathbb{P}_{z}\left(\tau_{O}^{N}<\theta_{\kappa_{1}} ;\left|\tilde{Z}^{N}\left(\tau_{O}^{N}\right)-y\right|<\delta\right) & =\mathbb{E}_{z}\left(\mathbb{P}_{z}\left(\tau_{O}^{N}<\theta_{\kappa_{1}} ;\left|\tilde{Z}^{N}\left(\tau_{O}^{N}\right)-y\right|<\delta \mid \mathcal{F}_{\sigma_{0}}\right)\right) \\
& =\mathbb{E}_{z}\left(\mathbb{P}_{\tilde{Z}^{N}\left(\sigma_{0}\right)}\left(\tau_{O}^{N}<\theta_{\kappa_{1}} ;\left|\tilde{Z}^{N}\left(\tau_{O}^{N}\right)-y\right|<\delta\right)\right) \\
& \geqslant \inf _{v \in \Gamma_{0}} \mathbb{P}_{v}\left(\tau_{O}^{N}<\theta_{\kappa_{1}} ;\left|\tilde{Z}^{N}\left(\tau_{O}^{N}\right)-y\right|<\delta\right)
\end{aligned}
$$

By definition of the $V_{\bar{O}}\left(z^{*}, y\right)$, there exists a curve $\varphi(t), 0 \leq t \leq T_{1}$ with $\varphi_{0}=z^{*}, \varphi_{T_{1}}=y$ such that

$$
I_{T_{1}}(\varphi) \leq V_{\bar{O}}\left(z^{*}, y\right)+\frac{\eta}{24}
$$

Moreover by Lemma $4.1 v \in \Gamma_{0}$ and $z^{*}$ can be connected by a curve $\tilde{\varphi}_{t}, 0 \leq t \leq T_{2}$ such that

$$
I_{T_{1}}(\tilde{\varphi})<\frac{\eta}{24}
$$

Combining $\tilde{\varphi}_{t}, 0 \leq t \leq T_{2}$ and $\varphi(t), 0 \leq t \leq T_{1}$, we construct a curve $\bar{\varphi}$ by

$$
\bar{\varphi}_{t}= \begin{cases}\tilde{\varphi}_{t} & \text { if } \quad t \in\left[0, T_{2}\right], \\ \varphi_{t-T_{2}} & \text { if } \quad t \in\left[T_{2}, T_{1}+T_{2}\right] .\end{cases}
$$

so that

$$
I_{T_{1}+T_{2}}(\bar{\varphi}) \leq V_{\bar{O}}\left(z^{*}, y\right)+\frac{\eta}{12}
$$

For this function $\bar{\varphi}$ we can choose $a$ such that its corresponding curve $\bar{\varphi}^{a}, 0 \leq t \leq T_{3}=T_{1}+T_{2}$ defined by

$$
\bar{\varphi}_{t}^{a}=(1-a) \bar{\varphi}_{t}+a z_{0}
$$


is such that $\operatorname{dist}\left(\bar{\varphi}_{t}^{a}, \widetilde{\partial O}\right) \geq c a$ and

$$
I_{T_{3}}\left(\bar{\varphi}^{a}\right) \leq I_{T_{3}}(\bar{\varphi})+\frac{\eta}{12}
$$

Thus

$$
I_{T_{3}}\left(\bar{\varphi}^{a}\right) \leq V_{\bar{O}}\left(z^{*}, y\right)+\frac{\eta}{6}
$$

According to the Lemma 4.1, there exist two functions $\varphi_{t}^{a, 1}, 0 \leq t \leq T^{a, 1}$ and $\varphi_{t}^{a, 2}, 0 \leq t \leq T^{a, 2}$ such that $\varphi_{0}^{a, 1}=v, \varphi_{T^{a, 1}}^{a, 1}=\varphi_{0}^{a}, \varphi_{0}^{a, 2}=\varphi_{T_{3}}^{a}, \varphi_{T^{a, 2}}^{a, 2}=y$ and

$$
I_{T^{a, 1}}\left(\varphi^{a, 1}\right) \leq \frac{\eta}{12} \quad \text { and } \quad I_{T^{a, 2}}\left(\varphi^{a, 2}\right) \leq \frac{\eta}{12} .
$$

Concatenating $\varphi_{t}^{a, 1}, 0 \leq t \leq T^{a, 1}, \bar{\varphi}_{t-T^{a, 1}}^{a}, T^{a, 1} \leq t \leq T_{3}+T^{a, 1}$ and $\phi_{t-T_{3}-T^{a, 1}}^{a, 2}, T_{3}+T^{a, 1} \leq t \leq T^{a, 2}+T_{3}+T^{a, 1}$, we obtain a function $\psi_{t}, 0 \leq t \leq T$ such that

$$
I_{T}(\psi) \leq V_{\bar{O}}\left(z^{*}, y\right)+\frac{\eta}{3}
$$

Let $\delta \leq \min \left\{\frac{1}{2} c a, \frac{\rho_{0}}{2}\right\}$ and define $G \subseteq D_{T, \bar{O}}$ to be the set of functions $\phi$ having the following properties:

- $\left|\phi_{T}-y\right|<\frac{1}{2} \delta$

- $\phi_{t}$ does not intersect $\left(\bar{O} \backslash O_{\frac{1}{4} \delta}\right) \backslash B_{\frac{1}{2} \delta}(y)$

- $\phi_{t}$ does not intersect $\overline{B_{\rho_{1}}\left(z^{*}\right)}$

$G$ is open and $\psi \in G$ (if $\psi$ intersect $\overline{B_{\rho_{1}}\left(z^{*}\right)}$ we use again Lemma 4.1 to modify it) we deduce from Theorem 3.2 and (4.26) that for large enough $N$

$$
\log \mathbb{P}_{v}^{N}(G) \geq-N\left(\inf _{\phi \in G, \phi_{0}=v} I_{T}(\varphi)+\frac{\eta}{3}\right) \geq-N\left(V_{\bar{O}}\left(z^{*}, y\right)+\frac{2 \eta}{3}\right)
$$

Moreover we remark that $\tilde{Z}^{N} \in G$ implies $\left|\tilde{Z}^{N}\left(\tau_{O}^{N} \wedge T\right)-y\right|<\frac{1}{2} \delta$ and $\theta_{\kappa_{1}}>T$. So for $N$ large enough,

$$
\log \mathbb{P}_{v}\left(\left|\tilde{Z}^{N}\left(\tau_{O}^{N} \wedge T\right)-y\right|<\frac{1}{2} \delta, \theta_{\kappa_{1}}>T\right) \geq-N\left(V_{\bar{O}}\left(z^{*}, y\right)+\frac{2 \eta}{3}\right)
$$

We have moreover

$$
\begin{aligned}
\Theta= & \mathbb{P}_{v}\left(\left|\tilde{Z}^{N}\left(\tau_{O}^{N}\right)-y\right|<\delta, \theta_{\kappa_{1}}>\tau_{O}^{N}\right) \\
\geq & \mathbb{P}_{v}\left(\left|\tilde{Z}^{N}\left(\tau_{O}^{N} \wedge T\right)-y\right|<\frac{1}{2} \delta,\left|\tilde{Z}^{N}\left(\tau_{O}^{N}\right)-y\right|<\delta, \theta_{\kappa_{1}}>\tau_{O}^{N}>T\right) \\
& +\mathbb{P}_{v}\left(\left|\tilde{Z}^{N}\left(\tau_{O}^{N} \wedge T\right)-y\right|<\frac{1}{2} \delta,\left|\tilde{Z}^{N}\left(\tau_{O}^{N}\right)-y\right|<\delta, \theta_{\kappa_{1}}>T \geq \tau_{O}^{N}\right) \\
& +\mathbb{P}_{v}\left(\left|\tilde{Z}^{N}\left(\tau_{O}^{N} \wedge T\right)-y\right|<\frac{1}{2} \delta,\left|\tilde{Z}^{N}\left(\tau_{O}^{N}\right)-y\right|<\delta, T \geq \theta_{\kappa_{1}}>\tau_{O}^{N}\right) \\
\geq & \Theta_{1}+\Theta_{2}+\Theta_{3} .
\end{aligned}
$$


Further

$$
\begin{aligned}
\Theta_{1} & =\mathbb{E}_{v}\left(\mathbb{P}_{v}\left(\left|\tilde{Z}^{N}\left(\tau_{O}^{N} \wedge T\right)-y\right|<\frac{1}{2} \delta, \theta_{\kappa_{1}}>\tau_{O}^{N}>T,\left|\tilde{Z}^{N}\left(\tau_{O}^{N}\right)-y\right|<\delta \mid \mathcal{F}_{T}\right)\right) \\
& =\mathbb{E}_{v}\left(\mathbb{P}_{v}\left(\left|\tilde{Z}^{N}\left(\tau_{O}^{N} \wedge T\right)-y\right|<\frac{1}{2} \delta, \theta_{\kappa_{1}}>\tau_{O}^{N}, \theta_{\kappa_{1}} \wedge \tau_{O}^{N}>T,\left|\tilde{Z}^{N}\left(\tau_{O}^{N}\right)-y\right|<\delta \mid \mathcal{F}_{T}\right)\right) \\
& =\mathbb{E}_{v}\left(\left|\tilde{Z}^{N}(T)-y\right|<\frac{1}{2} \delta, \theta_{\kappa_{1}} \wedge \tau_{O}^{N}>T, \mathbb{P}_{\tilde{Z}^{N}(T)}\left(\theta_{\kappa_{1}}>\tau_{O}^{N},\left|\tilde{Z}^{N}\left(\tau_{O}^{N}\right)-y\right|<\delta\right)\right) .
\end{aligned}
$$

Furthermore for $x$ such that $\operatorname{dist}(x, y)<\delta / 2$

$$
\mathbb{P}_{x}\left(\left|\tilde{Z}^{N}\left(\tau_{O}^{N}\right)-y\right|<\delta, \theta_{\kappa_{1}}>\tau_{O}^{N}\right) \geq \mathbb{P}_{x}\left(\tau_{O}^{N}<\gamma, \sup _{0 \leq t \leq \gamma}\left|\tilde{Z}^{N}(t)-y\right| \leq \delta\right),
$$

for all $\gamma>0$. In particular with $\gamma$ selected as in Lemma 4.16 and $\delta=\rho$, we deduce that

$$
\Theta_{1} \geq \mathbb{P}_{v}\left(\left|\tilde{Z}^{N}\left(\tau_{O}^{N} \wedge T\right)-y\right|<\frac{1}{2} \delta, \theta_{\kappa_{1}} \wedge \tau_{O}^{N}>T\right) \exp (-N \eta / 3)
$$

We also have

$$
\begin{aligned}
\Theta_{2} & =\mathbb{P}_{v}\left(\left|\tilde{Z}^{N}\left(\tau_{O}^{N} \wedge T\right)-y\right|<\frac{1}{2} \delta, \theta_{\kappa_{1}}>T \geq \tau_{O}^{N}\right) \\
& \geq \mathbb{P}_{v}\left(\left|\tilde{Z}^{N}\left(\tau_{O}^{N} \wedge T\right)-y\right|<\frac{1}{2} \delta, \theta_{\kappa_{1}}>T \geq \tau_{O}^{N}\right) \exp (-N \eta / 3)
\end{aligned}
$$

Thus since $\Theta_{3} \geq 0$,

$$
\begin{aligned}
\Theta \geq & \mathbb{P}_{v}\left(\left|\tilde{Z}^{N}\left(\tau_{O}^{N} \wedge T\right)-y\right|<\frac{1}{2} \delta, \theta_{\kappa_{1}} \wedge \tau_{O}^{N}>T\right) \exp (-N \eta / 3) \\
& +\mathbb{P}_{v}\left(\left|\tilde{Z}^{N}\left(\tau_{O}^{N} \wedge T\right)-y\right|<\frac{1}{2} \delta, \theta_{\kappa_{1}}>T \geq \tau_{O}^{N}\right) \exp (-N \eta / 3) \\
= & \mathbb{P}_{v}\left(\left|\tilde{Z}^{N}\left(\tau_{O}^{N} \wedge T\right)-y\right|<\frac{1}{2} \delta, \theta_{\kappa_{1}}>T\right) \exp (-N \eta / 3)
\end{aligned}
$$

It follows from (4.27) and (4.28) that

$$
\log \mathbb{P}_{v}\left(\left|\tilde{Z}^{N}\left(\tau_{O}^{N}\right)-y\right|<\delta, \theta_{\kappa_{1}}>\tau_{O}^{N}\right) \geq-N\left(V_{\bar{O}}\left(z^{*}, y\right)+\eta\right)
$$

Lemma 4.19. Given any $\eta$, there exists $\rho_{0}>0$ (which can be chosen arbitrarily small) such that for any $\rho_{2} \in\left(0, \rho_{0}\right)$, there exists $\rho_{1} \in\left(0, \rho_{2}\right)$ and $N_{\eta}$ such that for all $N>N_{\eta}$ and $z \in G_{0}^{1}$,

$$
\mathbb{P}_{z}\left(\tau_{O}^{N}<\theta_{\kappa_{1}}\right) \leq \exp \left(-N\left(V_{\widetilde{\partial O}}-\eta\right)\right) .
$$

Proof. Let $\delta>0$, we define

$$
\tau_{O_{\delta}}^{N}=\inf \left\{t>0: \tilde{Z}^{N, z}(t) \notin O_{\delta}\right\} .
$$


It is easy to see that $\tau_{O_{\delta}}^{N}<\tau_{O}^{N}$. Moreover if $\rho_{1}<\delta$, then $\tau_{O_{\delta}}^{N}<\theta_{\kappa_{1}}$ implies $\tau_{O_{\delta}}^{N}<\theta_{1}$. Thus $\forall z \in G_{0}^{1}$

$$
\mathbb{P}_{z}\left(\tau_{O}^{N}<\theta_{\kappa_{1}}\right) \leq \mathbb{P}_{z}\left(\tau_{O_{\delta}}^{N}<\theta_{\kappa_{1}}\right) \leq \mathbb{P}_{z}\left(\tau_{O_{\delta}}^{N}<\theta_{1}\right)
$$

Now we use the strong Markov property to write that $\forall z \in G_{0}^{1}$

$$
\mathbb{P}_{z}\left(\tau_{O_{\delta}}^{N}<\theta_{1}\right)=\mathbb{E}_{z}\left(\mathbb{P}_{\tilde{Z}^{N}\left(\sigma_{0}\right)}\left(\tau_{O_{\delta}}^{N}<\theta_{1}\right)\right)
$$

we deduce that

$$
\sup _{z \in G_{0}^{1}} \mathbb{P}_{z}\left(\tau_{O}^{N}<\theta_{\kappa_{1}}\right) \leqslant \sup _{v \in \Gamma_{0}} \mathbb{P}_{v}\left(\tau_{O_{\delta}}^{N}<\theta_{1}\right)
$$

Now, we establish that we can choose $\rho_{0}$ and $\delta$ sufficiently small such that for all $v \in \Gamma_{0}$ we have,

$$
\mathbb{P}_{v}\left(\tau_{O_{\delta}}^{N}<\theta_{1}\right) \leq \exp \left\{-N\left(V_{\widetilde{\partial O_{\delta}}}-\frac{2 \eta}{3}\right)\right\}
$$

Using Lemma 4.1 there exists $\rho>0$ such that $T(\rho)<\eta / 3 \mathcal{K}$. We take $\rho_{0}<\rho / 2, \delta$ and $\gamma$ sufficiently small such that for any trajectory $\phi_{t}, 0 \leq t \leq T$ starting from $v \in \Gamma_{0}$ and touching $O_{\delta} \backslash O_{\delta+\gamma}$ we have

$$
I_{T}(\phi) \geq V_{\widetilde{\partial O_{\delta}}}-\frac{\eta}{4}
$$

Moreover, using Lemma 4.5 there exists a constant $c$ and $T_{1}$ such that for all $N$ large enough, any $T>T_{1}$ and $v \in \overline{O_{\delta} \backslash G}$ where $G=\bigcup_{i=1}^{M} G_{i}^{1}$ we have

$$
\mathbb{P}_{v}\left(\tau \frac{N}{O_{\delta} \backslash G}>T\right) \leq \exp \left\{-N c\left(T-T_{1}\right)\right\}
$$

Now if we take any trajectory $\tilde{Z}^{N, v}$, with $v \in \Gamma_{0}$, and which reaches $O \backslash O_{\delta}$ before going to $G$ either spends time $T$ without touching $O \backslash O_{\delta}$ (the event $\left\{\tau \frac{N}{\bar{O}_{\delta} \backslash G}>T\right\}$ is realized) or reaches $O \backslash O_{\delta}$ before time $T$ and in this case the event

$$
\left\{\rho_{T}\left(\tilde{Z}^{N, v}, \Phi\left(V_{\widetilde{\partial O \delta}}-\frac{\eta}{4}\right)\right) \geq \gamma\right\} \text { is realized. }
$$

Hence, for all $v \in \Gamma_{0}$ we have from Lemma 4.5 and Theorem 3.2 (c)

$$
\begin{aligned}
\mathbb{P}_{v}\left(\tau_{O_{\delta}}^{N}<\theta_{1}\right) & \leq \mathbb{P}_{v}\left(\tau \frac{N}{O_{\delta} \backslash G}>T\right)+\mathbb{P}_{v}\left(\left\{\rho_{T}\left(\tilde{Z}^{N, v}, \Phi\left(V_{\widetilde{\partial O_{\delta}}}-\frac{\eta}{4}\right)\right) \geq \gamma\right\}\right) \\
& \leq \exp \left\{-N c\left(T-T_{1}\right)\right\}+\exp \left\{-N\left(V_{\widetilde{\partial O_{\delta}}}-\frac{\eta}{2}\right)\right\} \\
& \leq 2 \exp \left\{-N\left(V_{\widetilde{\partial O_{\delta}}}-\frac{\eta}{2}\right)\right\} \quad \text { taking } T \text { large enough } \\
& \leq \exp \left\{-N\left(V_{\widetilde{\partial O_{\delta}}}-\frac{3 \eta}{4}\right)\right\} \quad \text { taking } N \text { large enough such that } \frac{\ln (2)}{N}<\frac{\eta}{4}
\end{aligned}
$$

Moreover, $V_{\bar{O}}\left(z^{*},.\right)$ is continuous so if $\delta$ is sufficiently small then

$$
V_{\widetilde{\partial O_{\delta}}} \geq V_{\widetilde{\partial O}}-\frac{\eta}{4} .
$$


So

$$
\sup _{z \in G_{0}^{1}} \mathbb{P}_{z}\left(\tau_{O}^{N}<\theta_{\kappa_{1}}\right) \leq \exp \left\{-N\left(V_{\widetilde{\partial O}}-\eta\right)\right\}
$$

This prove the lemma.

\section{MAin Result on The EXIT MEASURE}

The proof of our main result, Theorem 5.3, will rely upon Lemma 5.2, for which the following notion will be essential.

Definition 5.1. Let $\mathcal{L}$ a subset of $\mathbb{N}$ and $W$ a subset of $\mathcal{L}$. A W-graph on $\mathcal{L}$ is an oriented graph which satisfies the following conditions

(a) It consists of arrows $i \rightarrow j, i \neq j$ with $i \in \mathcal{L} \backslash W$ and $j \in \mathcal{L}$.

(b) For all $i \in \mathcal{L} \backslash W$, there exists exactly one arrow such that $i$ is its initial point.

(c) For any $i \in \mathcal{L} \backslash W$ there exists a sequence of arrows leading from it to some point $j \in W$.

We will denote by $\operatorname{Gr}(W)$ the set of $W$-graphs and for $i \in \mathcal{L} \backslash W, j \in W$ we denote by $G r_{i, j}(W)$ the set of $W$-graphs in which the sequence of arrows leading from $i$ into $W$ ends at $j$. We can now show the analogue of Lemma 3.2 in [3].

Lemma 5.2. For all $y \in \widetilde{\partial O}$ and $\eta, \delta_{0}>0$ there exist $\rho, \delta<\delta_{0}$ and $N_{0}$, so that for all $z$ with $\operatorname{dist}\left(z, z^{*}\right)<\rho$ and $N>N_{0}$, we have

$$
\exp \left(-N\left(V_{\bar{O}}\left(z^{*}, y\right)-V_{\widetilde{\partial O}}+\eta\right)\right) \leq \mathbb{P}_{z}\left(\left|\tilde{Z}^{N}\left(\tau_{O}^{N}\right)-y\right|<\delta\right)
$$

and

$$
\mathbb{P}_{z}\left(\left|\tilde{Z}^{N}\left(\tau_{O}^{N}\right)-y\right|<\delta\right) \leq \exp \left(-N\left(V_{\bar{O}}\left(z^{*}, y\right)-V_{\widetilde{\partial O}}-\eta\right)\right)
$$

Proof. Let $y \in \widetilde{\partial O}$. We can always assume that $y \in \bigcup_{1}^{M} K_{i}$ else we add the compact $K=\{y\}$ in the list of the compacts and Assumption 2.1 remains true since $V_{O}(y, y)=0$ and If $y \mathcal{R} u$ for some $u \neq y$, then Lemma 4.3 implies that any $\omega$-limit point of (1.3) starting at $y$ is equivalent to $y$ and then $y$ was in a compact $K_{i}$.

In what follows we assume that $y \in K_{1}$. Let $\delta>0$. Using the strong Markov property we have, for all $z \in G_{0}^{1}$,

$$
\begin{aligned}
\mathbb{P}_{z}\left(\left|\tilde{Z}^{N}\left(\tau_{O}^{N}\right)-y\right|<\delta\right) \\
=\sum_{k=0}^{\infty} \mathbb{P}_{z}\left(\left|\tilde{Z}^{N}\left(\tau_{O}^{N}\right)-y\right|<\delta ; \theta_{\kappa_{k}} \leq \tau_{O}^{N}<\theta_{\kappa_{k+1}}\right) \\
=\sum_{k=0}^{\infty} \mathbb{E}_{z}\left[\mathbb{P}_{z}\left(\left|\tilde{Z}^{N}\left(\tau_{O}^{N}\right)-y\right|<\delta ; \theta_{\kappa_{k}} \leq \tau_{O}^{N}<\theta_{\kappa_{k+1}} \mid \mathcal{F}_{\theta_{\kappa_{k}}}\right)\right] \\
=\sum_{k=0}^{\infty} \mathbb{E}_{z}\left[\mathbb{P}_{z}\left(\left|\tilde{Z}^{N}\left(\tau_{O}^{N}\right)-y\right|<\delta ; \theta_{\kappa_{k}} \leq \tau_{O}^{N}<\theta_{\kappa_{k+1}} \mid Z^{N}\left(\theta_{\kappa_{k}}\right)\right)\right] \\
=\sum_{k=0}^{\infty} \mathbb{E}_{z}\left[\mathbb{P}_{\tilde{Z}^{N}\left(\theta_{\kappa_{k}}\right)}\left(\left|\tilde{Z}^{N}\left(\tau_{O}^{N}\right)-y\right|<\delta ; \tau_{O}^{N}<\theta_{\kappa_{1}}\right) ; \theta_{\kappa_{k}} \leq \tau_{O}^{N}\right] \\
=\sum_{k=0}^{\infty} \mathbb{E}_{z}\left[\mathbb{P}_{\tilde{Z}^{N}\left(\theta_{\kappa_{k}}\right)}\left(\left|\tilde{Z}^{N}\left(\tau_{O}^{N}\right)-y\right|<\delta \mid \tau_{O}^{N}<\theta_{\kappa_{1}}\right) \mathbb{P}_{\tilde{Z}^{N}\left(\theta_{\kappa_{k}}\right)}\left(\tau_{O}^{N}<\theta_{\kappa_{1}}\right) ; \theta_{\kappa_{k}} \leq \tau_{O}^{N}\right]
\end{aligned}
$$


Furthermore, $\forall z \in G_{0}^{1}$ we have

$$
\mathbb{P}_{z}\left(\left|\tilde{Z}^{N}\left(\tau_{O}^{N}\right)-y\right|<\delta \mid \tau_{O}^{N}<\theta_{\kappa_{1}}\right)=\frac{\mathbb{P}_{z}\left(\left|\tilde{Z}^{N}\left(\tau_{O}^{N}\right)-y\right|<\delta ; \tau_{O}^{N}<\theta_{\kappa_{1}}\right)}{\mathbb{P}_{z}\left(\tau_{O}^{N}<\theta_{\kappa_{1}}\right)}
$$

Let us now deduce the lower bound (5.1) from (5.4), (5.3) combined with Lemmas 4.18 and 4.19. Given $\eta, \delta>0$ pick $0<\rho_{1}<\rho_{0}$ satisfying both Lemmas with $\eta$ replaced by $\eta / 2$. For all $z \in G_{0}^{1}$ and $N$ large enough, we have

$$
\begin{aligned}
\frac{\mathbb{P}_{z}\left(\left|\tilde{Z}^{N}\left(\tau_{O}^{N}\right)-y\right|<\delta ; \tau_{O}^{N}<\theta_{\kappa_{1}}\right)}{\mathbb{P}_{z}\left(\tau_{O}^{N}<\theta_{\kappa_{1}}\right)} & \geq \frac{\exp \left(-N\left(V_{\bar{O}}\left(z^{*}, y\right)+\eta / 2\right)\right)}{\exp \left(-N\left(V_{\tilde{\partial O}}-\eta / 2\right)\right)} \\
& =\exp \left(-N\left(V_{\bar{O}}\left(z^{*}, y\right)-V_{\check{\partial O}}+\eta\right)\right)
\end{aligned}
$$

The lower bound (5.1) follows from this and (5.3).

For the upper bound (5.2), we first obtain a lower bound of the denominator of (5.4) as follows:

$$
\begin{aligned}
\mathbb{P}_{z}\left(\tau_{O}^{N}<\theta_{\kappa_{1}}\right) & =\mathbb{P}_{z}\left(\tilde{Z}_{1} \in \bigcup_{\ell=1}^{M} G_{\ell}^{1} ; \tilde{Z}^{N}\left(t^{-}\right)+\frac{1}{N} \sum_{j=1}^{k} h_{j} \triangle Q_{j}^{N}(t) \notin O \quad \text { for some } t \in\left[\theta_{1}, \theta_{2}\right)\right) \\
& =\mathbb{E}_{z}\left(\mathbb{P}_{\tilde{Z}_{1}}\left(\tau_{O}^{N}<\theta_{1}\right) ; \tilde{Z}_{1} \in \bigcup_{\ell=1}^{M} G_{\ell}^{1}\right)
\end{aligned}
$$

Now we use Lemma 4.16 to deduce that choosing $\gamma, \rho_{1}$ such that $0<\gamma<\eta, 0<\rho_{1}<\eta$ and $N_{0} \in \mathbb{N}$ we have for all $u \in \bigcup_{1}^{M} G_{\ell}^{1}$, $\operatorname{dist}(u, \widetilde{\partial O})<\rho_{1} / 2$ and then for $N>N_{0}$,

$$
\begin{aligned}
\mathbb{P}_{u}\left(\tau_{O}^{N}<\theta_{1}\right) & \geq \mathbb{P}_{u}\left(\tau_{O}^{N}<\gamma ; \sup _{0 \leq t \leq \gamma}\left|\tilde{Z}^{N}(t)-u\right|<\rho_{1}\right) \\
& \geq \exp (-N \eta)
\end{aligned}
$$

Thus, for all $\rho_{1}$ sufficiently small, $N$ large enough and all $z \in G_{0}^{1}$,

$$
\mathbb{P}_{z}\left(\tau_{O}^{N}<\theta_{\kappa_{1}}\right) \geq \exp (-N \eta / 4) \mathbb{P}_{z}\left(\tilde{Z}_{1} \in \bigcup_{1}^{M} G_{\ell}^{1}\right)
$$

By using the lemma 4.11 we have for all suitable small $\rho_{1}, \rho_{0}$, and $N$ large enough, and all $z \in G_{0}^{1}$,

$$
\mathbb{P}_{z}\left(\tilde{Z}_{1} \in \bigcup_{\ell=1}^{M} G_{\ell}^{1}\right) \geq \exp \left\{-N\left(V_{\tilde{\partial O}}+\eta / 4\right)\right\}
$$

We then deduce of (5.5) and (5.6) that

$$
\mathbb{P}_{z}\left(\tau_{O}^{N}<\theta_{\kappa_{1}}\right) \geq \exp \left(-N\left(V_{\partial O^{2}}+\eta / 2\right)\right)
$$


We now use the embedded chain $\tilde{Z}_{n}$ to obtain a upper bound of the numerator of (5.4) in the following way: given $\delta<\rho_{1}$ and $z \in G_{0}^{1}$,

$$
\begin{aligned}
\mathbb{P}_{z}\left(\left|\tilde{Z}^{N}\left(\tau_{O}^{N}\right)-y\right|<\delta ; \tau_{O}^{N}<\theta_{\kappa_{1}}\right) & \leq \mathbb{P}_{z}\left(\tilde{Z}^{N}\left(\tau_{O}^{N}\right) \in G_{1}^{1} ; \tau_{O}^{N}<\theta_{\kappa_{1}}\right) \\
& \leq \mathbb{P}_{z}\left(\tilde{Z}_{n} \in G_{1}^{1} \text { for some } 1 \leq n<\kappa_{1}\right) \\
& =\mathbb{E}_{z}\left(\mathbb{P}_{\tilde{Z}_{1}}\left(\tilde{Z}_{n} \in G_{1}^{1} \text { for some } 0 \leq n<\kappa_{1}\right)\right),
\end{aligned}
$$

where we assume of course that

$$
\mathbb{P}_{v}\left(\tilde{Z}_{n} \in G_{1}^{1} \text { for some } 0 \leq n<\kappa_{1}\right):=\left\{\begin{array}{ccc}
1 & \text { if } \quad v \in G_{1}^{1} \\
0 & \text { if } & v \in G_{0}^{1}
\end{array}\right.
$$

Now we try to have an upper bound of $\mathbb{P}_{v}\left(\tilde{Z}_{n} \in G_{1}^{1}\right.$ for some $\left.0 \leq n<\kappa_{1}\right)$ for $v \in G_{\ell}^{1}$ where $\ell \neq 0,1$. For all suitable $\rho_{1}, \rho_{0}$ and $N$ large we have for all $v \in G_{\ell}^{1}$

$$
\mathbb{P}_{v}\left(\tilde{Z}_{n} \in G_{1}^{1} \quad \text { for some } \quad 0 \leq n<\kappa_{1}\right)=q_{W}\left(v, G_{1}^{1}\right) .
$$

Where $q_{W}\left(v, G_{1}^{1}\right)$ is the probability that, starting from $v$ the Markov chain $\left(\tilde{Z}_{n}\right)$ hits $G_{1}^{1}$ when it first enters $G_{0}^{1} \cup G_{1}^{1}$.

Now we will use a result on the Markov chains described by [7] lemma 3.3 of chapter 6 in terms of the $W$-graphs on the sets $\mathcal{L}=\{0, \ldots, M\}$ where $W=\{0,1\}$. To apply this lemma we define the sets $X_{i}=G_{i}^{1}$ $\forall i \in\{0, \ldots, M\}$ and $X=\bigcup_{i=0}^{M} X_{i}$. If we define $a=\exp \left\{N \eta / 4^{M-1}\right\}$ and $p_{i, j}=\exp \left\{-N \widetilde{V}_{\bar{O}}\left(K_{i}, K_{j}\right)\right\}$, we deduce from lemma 4.9 that the assumptions of the lemma 3.3 of chapter 6 in [7] hold true, hence for all suitable $\rho_{1}$, $\rho_{0}$ and $N$ large enough, $v \in G_{\ell}^{1}$,

$$
q_{W}\left(v, G_{1}^{1}\right) \leq a^{4^{M-1}} \frac{\sum_{g \in G r_{\ell, 1}(W)} \prod_{(i \rightarrow j) \in g} p_{i, j}}{\sum_{g \in G r(W)} \prod_{(i \rightarrow j) \in g} p_{i, j}} .
$$

Thus

$$
q_{W}\left(v, G_{1}^{1}\right) \leq \exp \{N \eta / 7\} \frac{\sum_{g \in G r_{\ell, 1}(W)} \exp \left\{-N \sum_{(i \rightarrow j) \in g} \widetilde{V}_{\bar{O}}\left(K_{i}, K_{j}\right)\right\}}{\sum_{g \in G r(W)} \exp \left\{-N \sum_{(i \rightarrow j) \in g} \widetilde{V}_{\bar{O}}\left(K_{i}, K_{j}\right)\right\}} .
$$

It is easy to see that $\sum_{g \in G r_{\ell, 1}(W)} \exp \left\{-N \sum_{(i \rightarrow j) \in g} \widetilde{V}_{\bar{O}}\left(K_{i}, K_{j}\right)\right\}$ is equivalent to a positive constant $C_{1}$ which is the number of graphs in $G r_{\ell, 1}(W)$ at which the minimum of $\sum_{(i \rightarrow j) \in g} \widetilde{V}_{\bar{O}}\left(K_{i}, K_{j}\right)$ is attained multiplied by $\exp \left\{-N \min _{g \in G r_{\ell, 1}(W)} \sum_{(i \rightarrow j) \in g} \widetilde{V}_{\bar{O}}\left(K_{i}, K_{j}\right)\right\}$. We also see easily that the denominator in (5.10) is equivalent to a positive constant multiplied by $\exp \left\{-N \min _{g \in G r(W)} \sum_{(i \rightarrow j) \in g} \widetilde{V}_{\bar{O}}\left(K_{i}, K_{j}\right)\right\}$. Hence there exists $N_{0}$ such that for $N \geq N_{0}$

$$
q_{W}\left(v, G_{1}^{1}\right) \leq \exp \left\{-N\left(\min _{g \in G r_{\ell, 1}(W)} \sum_{(i, j) \in g} \widetilde{V}_{\bar{O}}\left(K_{i}, K_{j}\right)-\min _{g \in G r(W)} \sum_{(i, j) \in g} \widetilde{V}_{\bar{O}}\left(K_{i}, K_{j}\right)\right)+N \eta / 6\right\} .
$$


We remark here that in the case of a single attracting set $K_{0}, \widetilde{V}_{\bar{O}}\left(K_{i}, K_{0}\right)=0$ for all $i$. Then we have

$$
\min _{g \in G r(W)} \sum_{(i, j) \in g} \widetilde{V}_{\bar{O}}\left(K_{i}, K_{j}\right)=0
$$

We also have

$$
\min _{g \in G r_{\ell, 1}(W)} \sum_{(i, j) \in g} \widetilde{V}_{\bar{O}}\left(K_{i}, K_{j}\right)=V_{\bar{O}, K_{0}^{c}}\left(K_{\ell}, K_{1}\right)
$$

With these preceding remark, we deduce that

$$
\mathbb{P}_{v}\left(\tilde{Z}_{n} \in G_{1}^{1} \quad \text { for some } \quad 0 \leq n<\kappa_{1}\right) \leq \exp \left\{-N\left(V_{\bar{O}, K_{0}^{c}}\left(K_{\ell}, K_{1}\right)-\eta / 6\right)\right\} ; \quad v \in G_{\ell}^{1}, \quad \ell \neq 0,1 .
$$

Now according (5.9) and lemma 4.9 we have for $N>\frac{6 \log (M)}{\eta} \vee N_{0}$

$$
\begin{aligned}
& \mathbb{P}_{z}\left(\left|\tilde{Z}^{N}\left(\tau_{O}^{N}\right)-y\right|<\delta ; \tau_{O}^{N}<\theta_{\kappa_{1}}\right) \leq \mathbb{E}_{z}\left(\sum_{\ell=0}^{M} \mathbb{1}_{Z_{1} \in G_{\ell}^{1}} \mathbb{P}_{Z_{1}}\left(Z_{n} \in G_{1}^{1} \quad \text { for } \quad \text { some } 0 \leq n<\kappa_{1}\right)\right) \\
& =\mathbb{P}_{z}\left(\tilde{Z}_{1} \in G_{1}^{1}\right)+\sum_{\ell=2}^{M} \mathbb{E}_{z}\left(\mathbb{1}_{\tilde{Z}_{1} \in G_{\ell}^{1}} \mathbb{P}_{\tilde{Z}_{1}}\left(\tilde{Z}_{n} \in G_{1}^{1} \text { for some } 0 \leq n<\kappa_{1}\right)\right) \\
& \leq \exp \left\{-N\left(\widetilde{V}_{\bar{O}}\left(K_{0}, K_{1}\right)-\eta / 6\right)\right\}+\sum_{\ell=2}^{M} \exp \left\{-N\left(\widetilde{V}_{\bar{O}}\left(K_{0}, K_{\ell}\right)+V_{\bar{O}, K_{0}^{c}}\left(K_{\ell}, K_{1}\right)-\eta / 6\right)\right\} \\
& \leq \exp \left\{-N\left(\widetilde{V}_{\bar{O}}\left(K_{0}, K_{1}\right)-\eta / 6\right)\right\}+\exp \left\{-N\left(\min _{2 \leq \ell \leq M}\left\{\widetilde{V}_{\bar{O}}\left(K_{0}, K_{\ell}\right)+V_{\bar{O}, K_{0}^{c}}\left(K_{\ell}, K_{1}\right)\right\}-\eta / 3\right)\right\} \\
& \leq \exp \left\{-N\left(\widetilde{V}_{\bar{O}}\left(K_{0}, K_{1}\right) \wedge \min _{2 \leq \ell \leq M}\left\{\widetilde{V}_{\bar{O}}\left(K_{0}, K_{\ell}\right)+V_{\bar{O}, K_{0}^{c}}\left(K_{\ell}, K_{1}\right)\right\}-\eta / 2\right)\right\} .
\end{aligned}
$$

We remark here that

$$
\begin{aligned}
& \widetilde{V}_{\bar{O}}\left(K_{0}, K_{1}\right) \wedge \min _{2 \leq \ell \leq M}\left\{\widetilde{V}_{\bar{O}}\left(K_{0}, K_{\ell}\right)+V_{\bar{O}, K_{0}^{c}}\left(K_{\ell}, K_{1}\right)\right\} \\
& =V_{\bar{O}}\left(K_{0}, K_{1}\right) \\
& =V_{\bar{O}}\left(z^{*}, y\right)
\end{aligned}
$$

And then

$$
\mathbb{P}_{z}\left(\left|\tilde{Z}^{N}\left(\tau_{O}^{N}\right)-y\right|<\delta ; \tau_{O}^{N}<\theta_{\kappa_{1}}\right) \leq \exp \left\{-N\left(V\left(z^{*}, y\right)-\eta / 2\right)\right\}
$$

uniformly over $z \in G_{0}^{1}$, provided $\rho_{1}, \rho_{0}$ and $N$ are chosen in suitable way.

Combining (5.7), (5.11) and (5.4), we deduce that

$$
\mathbb{P}_{z}\left(\left|\tilde{Z}^{N}\left(\tau_{O}^{N}\right)-y\right|<\delta \mid \tau_{O}^{N}<\theta_{\kappa_{1}}\right) \leq \exp \left\{-N\left(V_{\bar{O}}\left(z^{*}, y\right)-V_{\partial \tilde{O}}-\eta\right)\right\}, \quad \text { for all } z \in G_{0}^{1},
$$

provided $\delta<\rho_{1}$ with $\rho_{1}$ sufficiently small and $N$ sufficiently large. As $\eta>0$ is arbitrary, we obtain the upper bound (5.2) from (5.3). This concludes the proof of lemma 5.2.

We finally establish our main result which is an analogue of Theorem 3.1 in [3]. 
Theorem 5.3. Assume that the Assumptions 2.1, 4.6 and 4.12 hold true. Then for $z \in O, y \in \widetilde{\partial O}$ and any $\eta>0, \delta_{0}>0$ there exist $\delta<\delta_{0}$ and $N_{0}$, such that for all $N>N_{0}$

$$
\exp \left(-N\left(S_{z}(y)+\eta\right)\right)<\mathbb{P}_{z}\left(\left|Z^{N}\left(\tau_{O}^{N}\right)-y\right|<\delta\right)<\exp \left(-N\left(S_{z}(y)-\eta\right)\right)
$$

where $S_{z}(y)$ is defined by:

$$
S_{z}(y)=V_{\bar{O}}(z, y) \wedge\left(V_{\bar{O}}\left(z^{*}, y\right)-V_{\widetilde{\partial O}}\right)
$$

Proof. So far we have worked with the reflected processes $\tilde{Z}^{N}$. We have come back to $Z^{N}$ in the above statement. Of course, the two processes are coupled by the fact that their equations are driven by the same Poisson processes. As a result, we can reformulate the definition (4.11) of $\tau_{O}^{N}$ as follows

$$
\mathbb{E} \tau_{O}^{N}=\inf \left\{t>0 ; Z^{N}(t) \in(\bar{O})^{c} \cup \widetilde{\partial O}\right\}
$$

which yields exactly the same stopping time. Also $\left|Z^{N}\left(\tau_{O}^{N}\right)-\tilde{Z}_{O}^{N}\left(\tau_{O}^{N}\right)\right| \leq N^{-1} \bar{h}$, where $\bar{h}=\sup _{1 \leq j \leq k}\left|h_{j}\right|$. So clearly the statement of the theorem is equivalent to the fact that for all $z \in O, y \in \widetilde{\partial O}$ and $\eta>0, \delta_{0}>0$ there exist $\delta<\delta_{0}$ and $N_{0}$, such that for all $N>N_{0}$,

$$
\exp \left(-N\left(S_{z}(y)+\eta\right)\right)<\mathbb{P}_{z}\left(\left|\tilde{Z}^{N}\left(\tau_{O}^{N}\right)-y\right|<\delta\right)<\exp \left(-N\left(S_{z}(y)-\eta\right)\right)
$$

We shall in fact establish the last statement.

We first remark that for $z=z^{*}$ the result is given by Lemma 5.2. If $z \in O \backslash\left\{z^{*}\right\}$, we make the restriction that $\rho_{0}$ be sufficiently small so that $\operatorname{dist}\left(z, K_{i}\right)>\rho_{0}$ for all $i=0, \ldots, M$. This allows us to write

$$
\begin{aligned}
\mathbb{P}_{z}\left(\left|\tilde{Z}^{N}\left(\tau_{O}^{N}\right)-y\right|<\delta\right)= & \mathbb{P}_{z}\left(\left|\tilde{Z}^{N}\left(\tau_{O}^{N}\right)-y\right|<\delta, \theta_{\kappa_{1}}<\tau_{O}^{N}\right) \\
& \left.+\mathbb{P}_{z}\left(\left|\tilde{Z}^{N}\left(\tau_{O}^{N}\right)-y\right|<\delta, \theta_{\kappa_{1}}>\tau_{O}^{N}\right)\right) \\
= & \mathbb{E}_{z}\left(\mathbb{P}_{\tilde{Z}_{\kappa_{1}}}\left(\left|\tilde{Z}^{N}\left(\tau_{O}^{N}\right)-y\right|<\delta\right) ; \theta_{\kappa_{1}}<\tau_{O}^{N}\right) \\
& +\mathbb{P}_{z}\left(\left|\tilde{Z}^{N}\left(\tau_{O}^{N}\right)-y\right|<\delta, \theta_{\kappa_{1}}>\tau_{O}^{N}\right) \\
= & \Pi_{1}+\Pi_{2} .
\end{aligned}
$$

We now split the rest of the proof in 5 steps.

Step 1. UPPER BOUnd of $\Pi_{1}$. Lemma 5.2 tells us that for all $z \in G_{0}^{1}$,

$$
\mathbb{P}_{z}\left(\left|\tilde{Z}^{N}\left(\tau_{O}^{N}\right)-y\right|<\delta\right) \leq \exp \left\{-N\left(V_{\bar{O}}\left(z^{*}, y\right)-V_{\widetilde{\partial O}}-\eta / 3\right)\right\}
$$

Hence $\Pi_{1}$ can be bounded from above as follows

$$
\begin{aligned}
\Pi_{1} & =\mathbb{E}_{z}\left(\mathbb{P}_{\tilde{Z}_{\kappa_{1}}}\left\{\left|\tilde{Z}^{N}\left(\tau_{O}^{N}\right)-y\right|<\delta\right\} ; \theta_{\kappa_{1}}<\tau_{O}^{N}\right) \\
& \left.\leq \mathbb{P}_{z}\left\{\theta_{\kappa_{1}}<\tau_{O}^{N}\right)\right\} \exp \left\{-N\left(V_{\bar{O}}\left(z^{*}, y\right)-V_{\widetilde{\partial O}}-\eta / 3\right)\right\} \\
& \leq \exp \left\{-N\left(V_{\bar{O}}\left(z^{*}, y\right)-V_{\widetilde{\partial O}}-\eta / 3\right)\right\}
\end{aligned}
$$


Step 2. Lower Bound of $\Pi_{1}$. From Lemma 4.10 we deduce that for suitably small $\rho_{0}, \rho_{1}$ there exists $N \in \mathbb{N}$ such that, for all $N>N_{0}$,

$$
\begin{aligned}
\mathbb{P}_{z}\left\{\theta_{\kappa_{1}}<\tau_{O}^{N}\right\} & \geq \mathbb{P}_{z}\left\{\tilde{Z}^{N}\left(\theta_{\kappa_{1}} \wedge \tau_{O}^{N}\right) \in G_{0}^{1}\right\} \\
& \geq \exp \{-N \eta / 2\}
\end{aligned}
$$

$\Pi_{1}$ can then be bounded from below as follows using Lemma 5.2

$$
\begin{aligned}
\Pi_{1} & =\mathbb{E}_{z}\left(\mathbb{P}_{\tilde{Z}_{\kappa_{1}}}\left(\left|\tilde{Z}^{N}\left(\tau_{O}^{N}\right)-y\right|<\delta\right), \theta_{\kappa_{1}}<\tau_{O}^{N}\right) \\
& \geq \mathbb{P}_{z}\left(\theta_{\kappa_{1}}<\tau_{O}^{N}\right) \exp \left\{-N\left(V_{\bar{O}}\left(z^{*}, y\right)-V_{\widetilde{\partial O}}+\eta / 2\right)\right\} \\
& \geq \exp \left\{-N\left(V_{\bar{O}}\left(z^{*}, y\right)-V_{\widetilde{\partial O}}+\eta\right)\right\} .
\end{aligned}
$$

Step 3. UpPeR BOUND of $\Pi_{2}$. We now obtain a upper bound for $\Pi_{2}$ by making the same computations as in the proof of lemma 5.2. Indeed

$$
\begin{aligned}
\mathbb{P}_{z}\left(\left|\tilde{Z}^{N}\left(\tau_{O}^{N}\right)-y\right|<\delta ; \tau_{O}^{N}<\theta_{\kappa_{1}}\right) & \leq \mathbb{P}_{z}\left(\tilde{Z}^{N}\left(\tau_{O}^{N}\right) \in G_{1}^{1} ; \tau_{O}^{N}<\theta_{\kappa_{1}}\right) \\
& \leq \mathbb{P}_{z}\left(\tilde{Z}_{n} \in G_{1}^{1} \text { for some } 0 \leq n \leq \kappa_{1}\right) \\
& =\mathbb{E}_{z}\left(\mathbb{P}_{\tilde{Z}_{1}}\left(\tilde{Z}_{n} \in G_{1}^{1} \text { for some } 0 \leq n<\kappa_{1}\right)\right) .
\end{aligned}
$$

Where we assume of course that,

$$
\mathbb{P}_{v}\left(\tilde{Z}_{n} \in G_{1}^{1} \quad \text { for some } \quad 0 \leq n<\kappa_{1}\right):=\left\{\begin{array}{lc}
1 & \text { if } \quad v \in G_{1}^{1} \\
0 & \text { if } \quad v \in G_{0}^{1}
\end{array}\right.
$$

For $v \in G_{\ell}^{1}, \ell \neq 0,1$ we can establish as in the proof of Lemma 5.2 that

$$
\mathbb{P}_{v}\left(\tilde{Z}_{n} \in G_{1}^{1} \quad \text { for some } \quad 0 \leq n<\kappa_{1}\right) \leqslant \exp \left\{-N\left(V_{\bar{O}, K_{0}^{c}}\left(K_{\ell}, K_{1}\right)-\eta\right)\right\}
$$

Hence

$$
\begin{aligned}
\Pi_{2} & =\mathbb{P}_{z}\left(\left|\tilde{Z}^{N}\left(\tau_{O}^{N}\right)-y\right|<\delta, \tau_{O}^{N}<\theta_{\kappa_{1}}\right) \\
& \leq \exp \left\{-N\left(\widetilde{V}_{\bar{O}}\left(z, K_{1}\right) \wedge \min _{2 \leq \ell \leq M}\left[\widetilde{V}_{\bar{O}}\left(z, K_{\ell}\right)+V_{\bar{O}, K_{0}^{c}}\left(K_{\ell}, K_{1}\right)\right]-2 \eta / 3\right)\right\} \\
& \leq \exp \left\{-N\left(V_{\bar{O}, K_{0}^{c}}(z, y)-2 \eta / 3\right)\right\} .
\end{aligned}
$$

Step 4. Lower Bound of $\Pi_{2}$. From Lemma 4.17, we deduce

$$
\begin{aligned}
\Pi_{2} & =\mathbb{P}_{z}\left(\left|\tilde{Z}^{N}\left(\tau_{O}^{N}\right)-y\right|<\delta, \theta_{\kappa_{1}}>\tau_{O}^{N}\right) \\
& \geq \exp \left\{-N\left(V_{\bar{O}, K_{0}^{c}}(z, y)+\eta\right)\right\} .
\end{aligned}
$$

Step 5. Conclusion. Thus, the term on the left in (5.14) can be bounded from above as follows, provided that $N>\frac{3 \log (2)}{\eta}$

$$
\begin{aligned}
& \mathbb{P}_{z}\left(\left|\tilde{Z}^{N}\left(\tau_{O}^{N}\right)-y\right|<\delta\right) \leq \exp \left\{-N\left(V_{\bar{O}}\left(z^{*}, y\right)-V_{\widetilde{\partial O}}-\eta / 3\right)\right\}+\exp \left\{-N\left(V_{\bar{O}, K_{0}^{c}}(z, y)-2 \eta / 3\right)\right\} \\
& \leq \exp \left\{-N\left(V_{\bar{O}, K_{0}^{c}}(z, y) \wedge\left[V_{\bar{O}}\left(z^{*}, y\right)-V_{\widetilde{\partial O}}\right]-\eta\right)\right\} .
\end{aligned}
$$


We now show that

$$
V_{\bar{O}, K_{0}^{c}}(z, y) \wedge\left[V_{\bar{O}}\left(z^{*}, y\right)-V_{\widetilde{\partial O}}\right]=V_{\bar{O}}(z, y) \wedge\left[V_{\bar{O}}\left(z^{*}, y\right)-V_{\widetilde{\partial O}}\right]
$$

Indeed we first remark that $V_{\bar{O}, K_{0}^{c}}(z, y) \geq V_{\bar{O}}(z, y)$. If $V_{\bar{O}, K_{0}^{c}}(z, y)>V_{\bar{O}}(z, y)$, then the nearly minimal paths for $V_{\bar{O}}(z, y)$ must come arbitrarily close to $K_{0}$, so

$$
V_{\bar{O}}(z, y)=V_{\bar{O}}\left(z, z^{*}\right)+V_{\bar{O}}\left(z^{*}, y\right)=V_{\bar{O}}\left(z^{*}, y\right),
$$

and then $V_{\bar{O}}\left(z^{*}, y\right)-V_{\widetilde{\partial O}}<V_{\bar{O}}(z, y)<V_{\bar{O}, K_{0}^{c}}(z, y)$ and (5.15) is true. This establishes the upper bound in (5.13).

In order to obtain the lower bound in (5.13), we deduce from the lower bounds for $\Pi_{1}$ and $\Pi_{2}$ that

$$
\begin{aligned}
\mathbb{P}_{z}\left(\left|\tilde{Z}^{N}\left(\tau_{O}^{N}\right)-y\right|<\delta\right) & \geq \exp \left\{-N\left(V_{\bar{O}}\left(z^{*}, y\right)-V_{\widetilde{\partial O}}+\eta\right)\right\}+\exp \left\{-N\left(V_{\bar{O}, K_{0}^{c}}(z, y)+\eta\right)\right\} \\
& \geq \exp \left\{-N\left(V_{\bar{O}, K_{0}^{c}}(z, y) \wedge\left[V_{\bar{O}}\left(z^{*}, y\right)-V_{\widetilde{\partial O}}\right]+\eta\right)\right\}
\end{aligned}
$$

and then the lower bound in (5.13) follows from (5.15).

We now deduce

Corollary 5.4. Assume that there exits a unique point $y^{*} \in \widetilde{\partial O}$ such that

$$
V_{\bar{O}}\left(z^{*}, y^{*}\right)=V_{\widetilde{\partial O}}=\inf _{y \in \widetilde{\partial O}} V_{\bar{O}}\left(z^{*}, y\right)
$$

then for all $\delta>0$, and $z \in O$

$$
\lim _{N \rightarrow \infty} \mathbb{P}_{z}\left(\left|Z^{N}\left(\tau_{O}^{N}\right)-y^{*}\right|<\delta\right)=1 .
$$

\section{Application to Four models}

In this section, we shall apply our result to 4 examples of epidemic models. In each of these models, for certain values of the parameters, the deterministic ODE model (which is the law of large numbers limit of our stochastic model) has a locally stable endemic equilibrium, i.e. an equilibrium with a positive proportion of infectious individuals (while a disease free equilibrium is an equilibrium with the proportion of infectious equal to zero). That equilibrium being stable, the ODE model predicts that the epidemic will persist for ever, hence the disease is endemic, this is why we call the corresponding equilibrium an endemic equilibrium. On the other hand, the stochastic model will hit soon or later the absorbing set of disease free states. Corollary 5.4 states that the preferred region of exit from the basin of attraction of the endemic equilibrium is the vicinity of the point $\bar{z}$ in $\widetilde{\partial O}$ which realizes the minimum of the function $V\left(z^{*}, z\right)$ on $\widetilde{\partial O}$, if that minimum is unique. We will show that in our 4 examples such uniqueness holds, and we will characterize the point $\bar{z}$.

In the two first examples, which satisfy condition $\mathbf{A}$ of Assumption 4.12, that is where $\widetilde{\partial O}=\left\{z, z_{1}=0\right\}, \bar{z}$ is the unique disease free equilibrium, which is an unstable equilibrium, which however is stable in the set $\left\{z_{1}=0\right\}$. In the two last examples, which satisfy condition $\mathbf{B}$ of Assumption 4.12, the boundary $\widetilde{\partial O}$ separates the basins of attraction of the endemic equilibrium $z^{*}$, and of the disease free equilibrium $z^{0}$. A third equilibrium, which is an unstable (in fact a hyperbolic) endemic equilibrium, attracts all points in $\widetilde{\partial O}$, that point is precisely $\bar{z}$ in those two examples.

In the four following models, we show that for $N$ large, with high probability the stochastic process hits the boundary of the basin or attraction of the endemic equilibrium near the point to which the law of large number limit converges, when restricted to this boundary. Note that $V_{\widetilde{\partial O}}$ is the value function of a deterministic optimal 
control problem. We will exploit Pontryagin's maximum principle, in order to prove the results of this section. We will first describe the relevant optimal control problem, and present Pontryagin's maximum principle in the case of that optimal control problem. Then we shall present the four models successively, and finally we shall prove our result. Note that Assumption 2.1 is easily verified in the first two examples, and has been carefully verified in [12] for the last two.

\subsection{Pontryagin maximum principle}

Let us formulate the optimal control problem, of which $V_{\widetilde{\partial O}}$ is the value function.

$B$ being the $d \times k$ matrix whose $j$-th column is the vector $h_{j}, 1 \leq j \leq k$, and $f: \mathbb{R}^{2}+\mapsto \mathbb{R}$ being defined by $f(a, b)=a \log (a / b)-a+b$, we consider the following optimal control problem

$$
(O C P)\left\{\begin{aligned}
\operatorname{Min}_{T>0, u \in L^{1}\left(0, T ; \mathbb{R}_{+}^{k}\right)} C(u), & \text { where } \\
C(u) & =\sum_{j=1}^{k} \int_{0}^{T} f\left(u_{j}(t), \beta_{j}\left(z_{t}\right)\right) \mathrm{d} t, \text { subject to the constraints } \\
\dot{z}_{t} & =B u_{t}, \quad z_{0}=z^{*}, \quad z_{T} \in M,
\end{aligned}\right.
$$

where $M:=\widetilde{\partial O}$ is the set of points $z \in A$ (or $\in A_{R}$ in the case of the SIR model with demography) which are such that $z_{t} \rightarrow \bar{z}$ as $t \rightarrow \infty$, if $z_{0}=z$ and $\dot{z}_{t}=b\left(z_{t}\right)=\sum_{j=1}^{k} \beta_{j}(z) h_{j}$. In other words, $\bar{z}$ is either the diseasefree equilibrium (in the situations considered in Sects. 6.2 and 6.3), or the unstable endemic equilibrium (in the situations considered in Sects. 6.4 and 6.5). The fact that $V_{\widetilde{\partial O}}$ coincides with the minimum of the cost functional $C(u)$ over the above set of admissible controls follows readily from the definition $(3.5)$ of $I_{T}(\phi)$, combined with the definition of $V_{\widetilde{\partial O}}$ which appears in the first lines of Section 4.

We associate to the above control problem the Hamiltonian

$$
H(z, r, u)=\langle r, B u\rangle-\sum_{j=1}^{k} f\left(u_{j}, \beta_{j}(z)\right) .
$$

The maximum principle states that (see $[13,17])$

Proposition 6.1. If $\left(\hat{T} ; \hat{u}_{t}, 0 \leq t \leq \hat{T}\right)$ is an optimal pair, then there exists an adjoint state $r \in C\left([0, \hat{T}] ; \mathbb{R}^{d}\right)$ such that

$$
\begin{aligned}
\dot{z}_{t} & =B \hat{u}_{t},: z_{0}=z^{*}, z_{\hat{T}} \in M, \\
\dot{r}_{t} & =\sum_{j=1}^{k}\left[\nabla \beta_{j}\left(z_{t}\right)-\hat{u}_{t}^{j} \frac{\nabla \beta_{j}\left(z_{t}\right)}{\beta_{j}\left(z_{t}\right)}\right], r_{\hat{T}} \perp M, \\
H\left(z_{t}, r_{t}, \hat{u}_{t}\right) & =\max _{v \in \mathbb{R}_{+}^{k}} H\left(z_{t}, r_{t}, v\right)=0,0 \leq t \leq \hat{T} .
\end{aligned}
$$

Note that $r_{\hat{T}} \perp M$ means that $r_{\hat{T}}$ and any tangent vector to $M$ at $z_{\hat{T}}$ are perpendicular. The fact that the Hamiltonian is zero at the final time is a consequence of two facts: the final time $T$ is not fixed and there is no final cost; the fact that the Hamiltonian is zero along the optimal trajectory then follows from the fact that it is constant, since none of the coefficient depends upon the variable $t$.

Let us exploit Proposition 6.1 to rewrite the system of ODEs for $z_{t}$ and $r_{t}$ along an optimal trajectory. We denote by $B^{*}$ the transposed of the matrix $B$. Since for each $1 \leq j \leq k, u \rightarrow\left(B^{*} r\right)_{j} u-f\left(u, \beta_{j}(z)\right)$ is concave, its maximum is achieved at the zero of its derivative, if it is non negative. We conclude that $\hat{u}_{j}=e^{\left(B^{*} r\right)_{j}} \beta_{j}(z)$. 
Consequently, along the optimal trajectory,

$$
\dot{z}_{t}=\sum_{j=1}^{k} e^{\left(B^{*} r_{t}\right)_{j}} \beta_{j}\left(z_{t}\right) h_{j}, \quad \dot{r}_{t}=\sum_{j=1}^{k}\left(1-e^{\left(B^{*} r_{t}\right)_{j}}\right) \nabla \beta_{j}\left(z_{t}\right) .
$$

Moreover, the optimal cost reads

$$
\hat{C}=\sum_{j=1}^{k} \int_{0}^{\hat{T}}\left(1-e^{\left(B^{*} r_{t}\right)_{j}}+\left(B^{*} r_{t}\right)_{j} e^{\left(B^{*} r_{t}\right)_{j}}\right) \beta_{j}\left(z_{t}\right) \mathrm{d} t .
$$

In the examples below, $d=2$. We shall denote by $x_{t}$ and $y_{t}$ the two components of $z_{t}$, and by $p_{t}$ and $q_{t}$ the two components of $r_{t}$.

\subsection{The SIRS model}

Let $x_{t}$ denote the proportion of infectious individuals in the population and $y_{t}$ the proportion of susceptibles. Since the total population size is constant, $1-x_{t}-y_{t}$ is the proportion of removed (also called recovered) individuals, who lose their immunity and become susceptible again at rate $\rho$. The deterministic SIRS model, see e.g. [2], can be written as

$$
\begin{aligned}
& \dot{x}_{t}=\lambda x_{t} y_{t}-\gamma x_{t}, \\
& \dot{y}_{t}=-\lambda x_{t} y_{t}+\rho\left(1-x_{t}-y_{t}\right) .
\end{aligned}
$$

The initial condition is supposed to satisfy $x_{0} \geq 0, y_{0} \geq 0, x_{0}+y_{0} \leq 1$. It is easy to see that the pair $\left(x_{t}, y_{t}\right)$ satisfies the same restrictions for all $t>0$. Here

$$
O=A=\left\{(x, y) \in \mathbb{R}_{+}^{2} ; x+y \leq 1\right\}, \quad \widetilde{\partial O}=\{0\} \times[0,1]
$$

We assume that the basic reproduction number $R_{0}=\lambda / \gamma$ satisfies $R_{0}>1$. Then there is a unique stable endemic equilibrium $z^{*}=\left(\frac{\rho}{\lambda} \frac{\lambda-\gamma}{\rho+\gamma}, \frac{\gamma}{\lambda}\right)$. The disease free equilibrium is $\bar{z}=(0,1)$.

The corresponding SDE is of the form (1.1), with $d=2, k=3, h_{1}=\left(\begin{array}{c}1 \\ -1\end{array}\right), h_{2}=\left(\begin{array}{c}-1 \\ 0\end{array}\right), h_{3}=\left(\begin{array}{l}0 \\ 1\end{array}\right)$, $\beta_{1}(x, y)=\lambda x y, \beta_{2}(x, y)=\gamma x, \beta_{3}(x, y)=\rho(1-x-y)$.

Note that in this example and in the next one, we do not need the definition of the reflected process $\tilde{Z}_{t}^{N}$, since it is identical to $Z_{t}^{N}$.

The system of ODES for the state and adjoint state (6.1) reads in this case

$$
\begin{aligned}
\dot{x}_{t} & =\lambda e^{p_{t}-q_{t}} x_{t} y_{t}-\gamma e^{-p_{t}} x_{t}, \\
\dot{y}_{t} & =-\lambda e^{p_{t}-q_{t}} x_{t} y_{t}+\rho e^{q_{t}}\left(1-x_{t}-y_{t}\right), \\
\dot{p}_{t} & =\lambda\left(1-e^{p_{t}-q_{t}}\right) y_{t}+\gamma\left(1-e^{-p_{t}}\right)-\rho\left(1-e^{q_{t}}\right), \\
\dot{q}_{t} & =\lambda\left(1-e^{p_{t}-q_{t}}\right) x_{t}-\rho\left(1-e^{q_{t}}\right), \\
x_{0} & =\frac{\rho}{\lambda} \frac{\lambda-\gamma}{\rho+\gamma}, y_{0}=\frac{\gamma}{\lambda}, x_{T}=0, q_{T}=0 .
\end{aligned}
$$




\subsection{The $S I R$ model with demography}

The deterministic SIR model with demography, see e.g. [2], can be written as

$$
\begin{aligned}
& \dot{x}_{t}=\lambda x_{t} y_{t}-(\gamma+\mu) x_{t} \\
& \dot{y}_{t}=-\lambda x_{t} y_{t}+\mu-\mu y_{t} .
\end{aligned}
$$

Here $x_{t}$ (resp. $y_{t}$ ) denotes the proportion of infectious (resp. susceptible) individuals in the population, if the initial condition $\left(x_{0}, y_{0}, u_{0}\right)$ satisfies $x_{0}, y_{0}, u_{0} \geq 0, x_{0}+y_{0}+u_{0}=1$. Note that $u_{t}$ denotes the proportion of recovered individuals in the population, which satisfies

$$
\dot{u}_{t}=\gamma x_{t}-\mu u_{t} .
$$

However, in the stochastic model, the coordinates of the vector $Z_{t}^{N}$ are not exact proportions, since we divide the number of individuals in each compartment by $N$, while the total population $N_{t}$ fluctuates around $N$. This is why $Z_{t}^{N}$ lives in $\mathbb{R}_{+}^{2}$, and

$$
O=A=\mathbb{R}_{+}^{2}, \quad \widetilde{\partial O}=\{0\} \times \mathbb{R}_{+} .
$$

As opposed to the SIRS model, the removed individuals do not loose their immunity, rather new susceptibles are born at rate $\mu$, which is the rate at which both susceptibles and infectious die (the infectious heal at rate $\rho$ as in the $S I R S$ model). If $R_{0}=\frac{\lambda}{\gamma+\mu}>1$, there is a stable endemic equilibrium $z^{*}=\left(\frac{\mu}{\gamma+\mu}-\frac{\mu}{\lambda}, \frac{\gamma+\mu}{\lambda}\right)$, and a disease free equilibrium $\bar{z}=(0,1)$.

The corresponding SDE is of the form (1.1), with $d=2, k=4, h_{1}=\left(\begin{array}{c}1 \\ -1\end{array}\right), h_{2}=\left(\begin{array}{c}-1 \\ 0\end{array}\right), h_{3}=\left(\begin{array}{l}0 \\ 1\end{array}\right)$, $h_{4}=\left(\begin{array}{c}0 \\ -1\end{array}\right), \beta_{1}(x, y)=\lambda x y, \beta_{2}(x, y)=(\gamma+\mu) x, \beta_{3}(x, y)=\mu, \beta_{4}(x, y)=\mu y$. One can show, see [2], that the cost needed to hit the boundary $\{x+y=R\}$ tends to $\infty$ as $R \rightarrow \infty$, hence for $R$ large enough, if we restrict ourself to the subset $A_{R}=\left\{(x, y) \in \mathbb{R}_{+}^{2}, x+y \leq R\right\}, \min _{z \in \partial A_{R}} V\left(z^{*}, z\right)=\min _{z_{1}=0} V\left(z^{*}, z\right)$. Also $A_{R}$ is not exactly $A_{1}$ which has been considered so far, it is easily seen that all our results extend to this new situation, and Corollary 5.4 combined with Proposition 6.2 tells us that for large $R$, the process $Z^{N}$ hit $\left\{z_{1}=0\right\} \cup\{|z| \geq R\}$ near a given point of the boundary $\left\{z_{1}=0\right\}$ which is independent of $R$, with probability close to 1 .

The system of ODEs for the state and adjoint state (6.1) reads in this case

$$
\begin{aligned}
\dot{x}_{t} & =\lambda e^{p_{t}-q_{t}} x_{t} y_{t}-(\gamma+\mu) e^{-p_{t}} x_{t}, \\
\dot{y}_{t} & =-\lambda e^{p_{t}-q_{t}} x_{t} y_{t}+\mu e^{q_{t}}-\mu e^{-q_{t}} y_{t}, \\
\dot{p}_{t} & =\lambda\left(1-e^{p_{t}-q_{t}}\right) y_{t}+(\gamma+\mu)\left(1-e^{-p_{t}}\right), \\
\dot{q}_{t} & =\lambda\left(1-e^{p_{t}-q_{t}}\right) x_{t}+\mu\left(1-e^{-q_{t}}\right), \\
x_{0} & =\frac{\mu}{\gamma+\mu}-\frac{\mu}{\lambda}, y_{0}=\frac{\gamma+\mu}{\lambda}, x_{T}=0, q_{T}=0 .
\end{aligned}
$$

\subsection{The $S I V$ model}

In this model, some of the individuals are vaccinated. Also the vaccine is not perfect, it gives a partial protection. If we denote by $x_{t}$ (resp. $y_{t}$ ) the proportion of infectious (resp. of vaccinated) individuals at time $t$, 


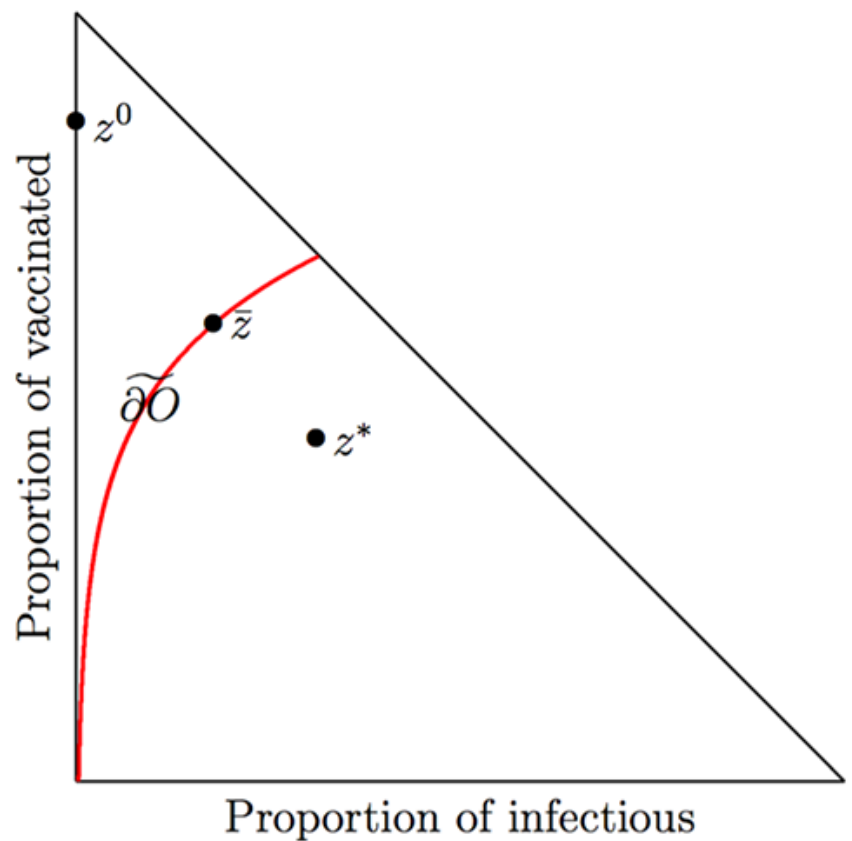

Figure 1. The 3 equilibria and $\widetilde{\partial O}$ in case of the SIV model.

the model studied by [9] reads

$$
\begin{aligned}
& \dot{x}_{t}=(\beta-\mu-\gamma) x_{t}-\beta(1-\chi) x_{t} y_{t}-\beta x_{t}^{2}, \\
& \dot{y}_{t}=\eta\left(1-x_{t}\right)-(\eta+\mu+\theta) y_{t}-\chi \beta x_{t} y_{t} .
\end{aligned}
$$

Here

$$
A=\left\{(x, y) \in \mathbb{R}_{+}^{2} ; x+y \leq 1\right\} .
$$

For certain values of the parameters, it is shown that this model has one disease-free equilibrium, one locally stable endemic equilibrium $z^{*}$, and a third equilibrium $\bar{z}$ which lies on the characteristic boundary which separates the basins of attraction of the two other equilibria, which is here $\widetilde{\partial O}, O$ being the basin of attraction of $z^{*}$. The corresponding SDE is of the form $(1.1)$, with $d=2, k=7, h_{1}=\left(\begin{array}{l}1 \\ 0\end{array}\right), h_{2}=\left(\begin{array}{c}1 \\ -1\end{array}\right), h_{3}=\left(\begin{array}{c}-1 \\ 0\end{array}\right)$, $h_{4}=\left(\begin{array}{c}0 \\ -1\end{array}\right), h_{5}=\left(\begin{array}{l}0 \\ 1\end{array}\right), h_{6}=\left(\begin{array}{c}-1 \\ 0\end{array}\right)$ and $h_{7}=\left(\begin{array}{c}0 \\ -1\end{array}\right), \beta_{1}(x, y)=\beta x(1-x-y), \beta_{2}(x, y)=\chi \beta x y, \beta_{3}(x, y)=\gamma x$, $\beta_{4}(x, y)=\theta y, \beta_{5}(x, y)=\eta(1-x-y), \beta_{6}(x, y)=\mu x, \beta_{7}(x, y)=\mu y$.

The system of ODES for the state and adjoint state (6.1) reads in this case

$$
\begin{aligned}
\dot{x}_{t} & =\beta e^{p_{t}} x_{t}\left(1-x_{t}-y_{t}\right)+\chi \beta e^{p_{t}-q_{t}} x_{t} y_{t}-(\gamma+\mu) e^{-p_{t}} x_{t}, \\
\dot{y}_{t} & =-\chi \beta e^{p_{t}-q_{t}} x_{t} y_{t}-(\theta+\mu) e^{-q_{t}} y_{t}+\eta e^{q_{t}}\left(1-x_{t}-y_{t}\right), \\
\dot{p}_{t} & =\beta\left(1-e^{p_{t}}\right)\left(1-2 x_{t}-y_{t}\right)+\chi \beta\left(1-e^{p_{t}-q_{t}}\right) y_{t}+\gamma\left(1-e^{-p_{t}}\right)-\eta\left(1-e^{q_{t}}\right)+\mu\left(1-e^{-p_{t}}\right), \\
\dot{q}_{t} & =-\beta\left(1-e^{p_{t}}\right) x_{t}+\chi \beta\left(1-e^{p_{t}-q_{t}}\right) x_{t}+\theta\left(1-e^{-q_{t}}\right)+\eta\left(e^{q_{t}}-1\right)+\mu\left(1-e^{-q_{t}}\right), \\
x_{0} & =x^{*}, y_{0}=y^{*},\left(x_{T}, y_{T}\right) \in M,\left(p_{T}, q_{T}\right) \perp M .
\end{aligned}
$$




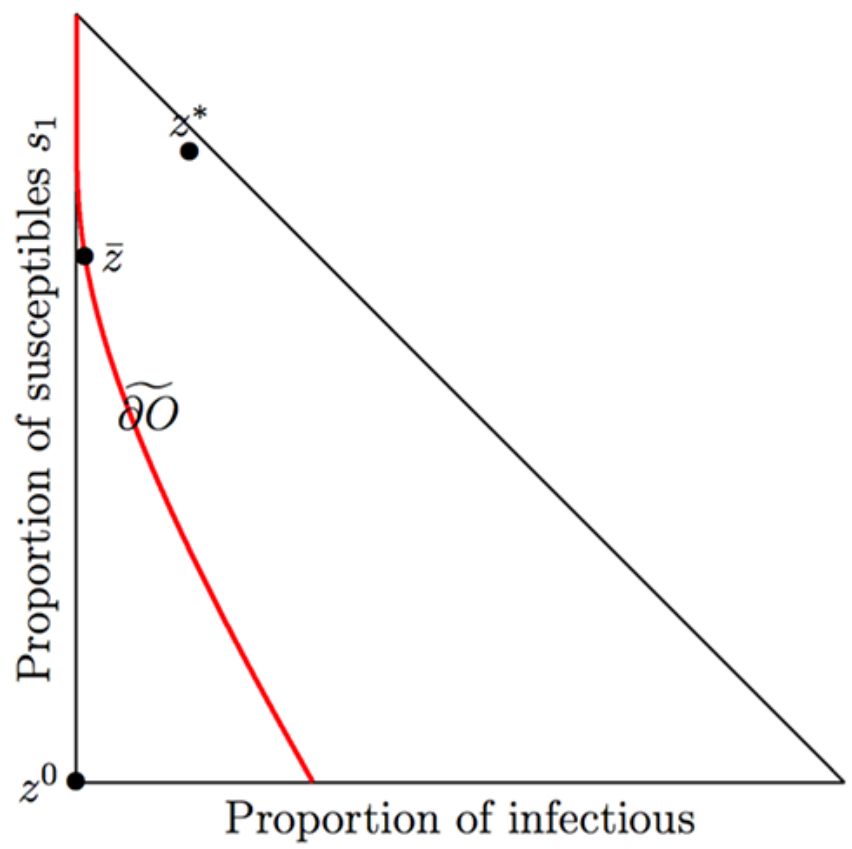

Figure 2. The 3 equilibria and $\widetilde{\partial O}$ in case of the $S_{0} I S_{1}$ model.

\subsection{The $S_{0} I S_{1}$ model}

This is a version of the $S I R$ model, where the recovered individuals are susceptible, but with a susceptibility which is less that that of those who have never been infected. They are of type $S_{1}$. This model has been studied in [14]. Let $x_{t}$ (resp. $y_{t}$ ) denote the proportion of infectious (resp. of type $S_{1}$ ) individuals. The ODE reads

$$
\begin{aligned}
\dot{x}_{t} & =\beta\left(1-x_{t}-y_{t}\right) x_{t}-(\mu+\alpha) x_{t}+r \beta x_{t} y_{t}, \\
\dot{y}_{t} & =\alpha x_{t}-\mu y_{t}-r \beta x_{t} y_{t} .
\end{aligned}
$$

Here

$$
A=\left\{(x, y) \in \mathbb{R}_{+}^{2} ; x+y \leq 1\right\}
$$

Again for certain values of the parameters, we have the same large time description as for the $S I V$ model, and $\widetilde{\partial O}$ is the characteristic boundary which separates the basins of attraction of the two local stable equilibria, $O$ being the basin of attraction of $z^{*}$. The corresponding SDE is of the form (1.1), with $d=2, k=5, h_{1}=\left(\begin{array}{l}1 \\ 0\end{array}\right)$, $h_{2}=\left(\begin{array}{c}-1 \\ 1\end{array}\right), h_{3}=\left(\begin{array}{c}-1 \\ 0\end{array}\right), h_{4}=\left(\begin{array}{c}1 \\ -1\end{array}\right)$ and $h_{5}=\left(\begin{array}{c}0 \\ -1\end{array}\right), \beta_{1}(x, y)=\beta x(1-x-y), \beta_{2}(x, y)=\alpha x, \beta_{3}(x, y)=\mu x$, $\beta_{4}(x, y)=r \beta x y$ and $\beta_{5}(x, y)=\mu y$.

The system of ODES for the state and adjoint state (6.1) reads in this case

$$
\begin{aligned}
& \dot{x}_{t}=\beta e^{p_{t}} x_{t}\left(1-x_{t}-y_{t}\right)-\alpha e^{q_{t}-p_{t}} x_{t}-\mu e^{-p_{t}} x_{t}+r \beta e^{p_{t}-q_{t}} x_{t} y_{t}, \\
& \dot{y}_{t}=\alpha e^{q_{t}-p_{t}} x_{t}-r \beta e^{p_{t}-q_{t}} x_{t} y_{t}-\mu e^{-q_{t}} y_{t}, \\
& \dot{p}_{t}=\beta\left(1-e^{p_{t}}\right)\left(1-2 x_{t}-y_{t}\right)+\alpha\left(1-e^{q_{t}-p_{t}}\right)+\mu\left(1-e^{-p_{t}}\right)+r \beta\left(1-e^{p_{t}-q_{t}}\right) y_{t},
\end{aligned}
$$




$$
\begin{aligned}
\dot{q}_{t} & =-\beta\left(1-e^{p_{t}}\right) x_{t}+r \beta\left(1-e^{p_{t}-q_{t}}\right) x_{t}+\mu\left(1-e^{-q_{t}}\right), \\
x_{0} & =x^{*}, y_{0}=y^{*},\left(x_{T}, y_{T}\right) \in M,\left(p_{T}, q_{T}\right) \perp M .
\end{aligned}
$$

\subsection{The result}

Recall that we start our process $Z_{t}^{N}$ from the endemic equilibrium, i.e. $Z_{0}^{N}=z^{*}$. Since is $N$ assumed to be large, $Z_{t}^{N}$ stays for a long time close to its law of large numbers limit, hence close to $z^{*}$. Wentzell-Freidlin's theory of "small random perturbations of dynamical systems" suggests that at some large time, which is of the order of $\exp \left(N V_{\widetilde{\partial O}}\right), Z_{t}^{N}$ will hit the boundary $\widetilde{\partial O}$. In the two first examples where $\widetilde{\partial O}=\left\{z_{1}=0\right\}$, at that time the epidemic stops, we say that we have extinction of the endemic disease. In fact the same is true in the last two examples, since as soon as the process crosses the boundary $\widetilde{\partial O}$, it converges in time of order one to the disease free equilibrium. Consequently the asymptotic of the time to hit or cross that boundary (which is a.s. the same event) is the same as the one for the epidemic to go extinct.

When on the boundary $\widetilde{\partial O}$, the solution of the ODE converges to $\bar{z}$. Recalling the notations defined at the beginning of Section 4 , it clearly follows that $V_{\widetilde{\partial O}}=V_{\bar{O}}\left(z^{*}, \bar{z}\right)$. What we want to show is that this minimum is unique, i.e.

Proposition 6.2. In each of the above four examples, for all $z \in \widetilde{\partial O} \backslash\{\bar{z}\}, V_{\bar{O}}\left(z^{*}, \bar{z}\right)<V_{\bar{O}}\left(z^{*}, z\right)$.

This shows, thanks to Corollary 5.4, that for large $N, Z^{N, z}$ will exit the domain of attraction of the endemic equilibrium $z^{*}$ in the vicinity of $\bar{z}$ with probability close to 1 .

We now turn to the

Proof of Proposition 6.2 Our assumptions are easy to verify in the first two examples, the verification in the last two examples has been done in [12]. We consider the optimal control problem which has been stated in subsection 6.1, in one of the four above examples. Suppose first that there exists a minimizing sequence $\left\{u_{n}\right.$, $n \geq 1\} \subset L^{1}\left(\mathbb{R}_{+} ; \mathbb{R}_{+}^{k}\right)$ such that the corresponding trajectory $z_{t}$ hits the target $M$ at some point $z_{n} \in \widetilde{\partial O} \backslash\{\bar{z}\}$ in time $T_{n}$, with $T:=\sup _{n} T_{n}<\infty$. Since $I_{T}$ is a good rate function (i.e. its level sets are compact), see Theorem 3 in [11], there exists a subsequence $z_{n_{k}}$ which converges to a optimal trajectory $\hat{z}$ which hits $M$ at point $z \neq \bar{z}$ at time $\hat{T} \leq T$, with a control $\hat{u} \in L^{1}\left([0, T] ; \mathbb{R}_{+}^{k}\right)$. We concatenate $\hat{z}$ with the solution of the ODE starting from $z$, which converges to $\bar{z}$ (in infinite time). Since the second part of the trajectory runs at no cost, the whole trajectory is optimal for the same control problem as above, but with the constraint that $\bar{z}$ must be the final point. We apply the Pontryagin maximum principle to this new optimal control problem, which implies the existence of a continuous adjoint state $\left(p_{t}, q_{t}\right)$. Since $p_{t}=q_{t}=0$ for $t>\hat{T}$, we have $p_{\hat{T}}=q_{\hat{T}}=0$. But this is not possible. $z_{t}$ being bounded, the solution $\left(p_{t}, q_{t}\right)$ of the adjoint state equation cannot hit $(0,0)$ in finite time. One way to see this is to note that the function $\left(p_{t}, q_{t}\right)$ time reversed from time $\hat{T}$ would solve an ODE starting from $(0,0)$, whose unique solution is $\left(p_{t}, q_{t}\right) \equiv(0,0)$, see the second equation in (6.1). We conclude from the above argument that if an optimal trajectory converges to some point $z \neq \bar{z}$, then it does so in infinite time. Consequently $\left(x_{\infty}, y_{\infty}, p_{\infty}, q_{\infty}\right)$ must be a fixed point of $(6.1)$. It remains to show that $(\bar{x}, \bar{y}, 0,0)$ is the only admissible fixed point. The argument is now slightly different in the various considered examples.

In the first two examples (SIRS and $S I R$ with demography), we know that $x_{\infty}$ (the first coordinate of $z_{\infty}$ ) and the second coordinate $q_{\infty}$ of $r_{\infty}$ vanish. Consequently $y_{\infty}$ must be the zero of $1-y$, hence equals 1 .

In the two other cases, we first note that both $p_{\infty}$ and $q_{\infty}$ must be finite. Indeed, either the solution must remain bounded, or else would explode in finite time, which contradicts the existence of the adjoint state on $[0,+\infty)$. We next show that both coordinates of $z_{\infty}, x_{\infty}$ and $y_{\infty}$, are positive. In case of the $S I V$ model, we first note that $y_{\infty}=0$ would imply $x_{\infty}=1$, but $(1,0)$ is clearly not on $M$. On the other hand, $x_{\infty}=0$ would imply that the second coordinate $q_{\infty}$ of $r_{\infty}$ vanishes, and $y_{\infty}=\frac{\eta}{\eta+\mu+\theta}$, which again gives a point not on $M$. In case of the $S_{0} I S_{1}$ model, we note that $x_{\infty}=0$ implies $y_{\infty}=0$, and the reserve implication is also true, but $(0,0)$ is not on $M$. Finally, since $\hat{C}<\infty$, the running cost must converge to 0 as $t \rightarrow \infty$. For each $1 \leq j \leq k$ such that $\beta_{j}\left(z_{\infty}\right)>0$, this implies that $\left\langle r_{t}, h_{j}\right\rangle \rightarrow 0$ as $t \rightarrow \infty$. This is true for $j=3$ and 4 in case of the $S I V$ 
model, for $j=3$ and 5 in case of the $S_{0} I S_{1}$ model. In both cases, it implies that $\left(p_{\infty}, q_{\infty}\right)=(0,0)$. Consequently $z_{\infty}$ is a zero of $b(z)=\sum_{j=1}^{k} \beta_{j}(z) h_{j}$ and belongs to $M$, hence equals $\bar{z}$.

We next need to consider the case where all minimizing sequences $\left\{u_{n}, n \geq 1\right\}$ satisfy $T_{n} \rightarrow \infty$, as $n \rightarrow \infty$. The limiting trajectory, which is optimal, reaches the target $M$ in infinite time. The argument just developed shows that the point of $M$ to which the optimal trajectory converges must be $z_{\infty}=\bar{z}$.

We have proved that, if $z_{\text {inf }}$ is such that $V\left(z^{*}, z_{\text {inf }}\right)=\min _{z \in \widetilde{\partial O}} V\left(z^{*}, z\right)$, then $z_{\text {inf }}=\bar{z}$. Hence for any $z \in$ $\widetilde{\partial O} \backslash\{\bar{z}\}, V\left(z^{*}, z\right)>V\left(z^{*}, \bar{z}\right)$, and the result is established.

Acknowledgements. The authors want to thank an anonymous Referee for his careful reading of our paper, and his many remarks and criticisms, which helped us to improve significantly our earlier version.

\section{REFERENCES}

[1] K.B. Athreya and P.E. Ney, Branching Processes. Vol. 196 of Grundlehren der mathematischen Wissenschaften. Springer (1972).

[2] T. Britton and E. Pardoux, Stochastic epidemics in a homogeneous community. Part I of Stochastic Epidemic Models with Inference, edited by T. Britton and E. Pardoux. Vol. 2255 of Lecture Notes in Math. Springer (2019) 1-120.

[3] M.V. Day, Large deviations results for the exit problem with characteristic boundary. J. Math. Anal. Appl. 147 (1990) $134-153$.

[4] A. Dembo and O. Zeitouni, Large deviations techniques and applications, Vol. 38 of Appplications of Mathematics. Springer (2009).

[5] H. Doss and P. Priouret, Petites perturbations de systèmes dynamiques avec réflexion, in Séminaire de Probabilités XVII. Vol. 986 of Lecture Notes in Math. Springer (1983) 353-370.

[6] S.N. Ethier and T.G. Kurtz, Markov Processes, Characterization and Convergence. J. Wiley \& Sons, Inc. (1986).

[7] M.I. Freidlin and A.D. Wentzell, Random perturbations of dynamical systems, Vol. 260 of Grundlehren der mathematischen Wissenschaften. Springer (2012).

[8] P. Kratz and E. Pardoux, Large deviations for infectious diseases models, Chapter 7 of Séminaire de Probabilités XLIX. Vol. 2215 of Lecture Notes in Math.. Springer (2018) 221-327.

[9] C.M. Kribs-Zaleta and J.X. Velasco-Hernandez, A simple vaccination model with multiple endemic states. Math. Biosci. 164 (2000) 183-201.

[10] T.G. Kurtz, Strong approximation theorems for density dependent Markov chains. Stoch. Process. Appl. 6 (1978) $223-240$.

[11] E. Pardoux and B. Samegni-Kepgnou, Large deviations principle for Poisson driven SDE in epidemic models. J. Appl. Probab. 54 (2017) 905-920.

[12] E. Pardoux and B. Samegni-Kepgnou, Large deviations principle for Reflected Poisson driven SDEs in epidemic models. Stoch. Anal. Appl. 37 (2019) 836-864.

[13] L.S. Pontryagin, V.G. Boltyanskii, R.V. Gamkrelidze and E.F. Mishchenko, The mathematical theory of optimal processes. Transl. by K. N. Trirogoff. Edited by L. W. Neustadt. John Wiley \& Sons (1962).

[14] M. Safan, H. Heesterbeek and K. Dietz, The minimum effort required to eradicate infections in models with backward bifurcation. J. Math. Biol. 53 (2006) 703-718.

[15] A. Shwartz and A. Weiss, Large Deviations for Performance Analysis. Chapman Hall, London (1995).

[16] A. Shwartz and A. Weiss, Large deviations with diminishing rates. Math. Oper. Res. 30 (2005) 281-310.

[17] E. Trélat, Contrôle optimal: théorie \& applications. Vuibert (2008). 